\title{
Asymptotic geometry in products of Hadamard spaces with rank one isometries
}

\author{
GABRIELE LINK
}

In this article we study asymptotic properties of certain discrete groups $\Gamma$ acting by isometries on a product $X=X_{1} \times X_{2}$ of locally compact Hadamard spaces which admit a geodesic without flat half-plane. The motivation comes from the fact that KacMoody groups over finite fields, which can be seen as generalizations of arithmetic groups over function fields, belong to the considered class of groups. Hence one may ask whether classical properties of discrete subgroups of higher rank Lie groups as in Benoist [5] and Quint [16] hold in this context.

In the first part of the paper we describe the structure of the geometric limit set of $\Gamma$ and prove statements analogous to the results of Benoist in [5]. The second part is concerned with the exponential growth rate $\delta_{\theta}(\Gamma)$ of orbit points in $X$ with a prescribed "slope" $\theta \in(0, \pi / 2)$, which appropriately generalizes the critical exponent in higher rank. In analogy to Quint's result in [16] we show that the homogeneous extension $\Psi_{\Gamma}$ to $\mathbb{R}_{\geq 0}^{2}$ of $\delta_{\theta}(\Gamma)$ as a function of $\theta$ is upper semicontinuous and concave.

20F69, 51F99; 53C23, 20G15, 22D40, 51E24

\section{Introduction}

Let $\left(X_{1}, d_{1}\right),\left(X_{2}, d_{2}\right)$ be Hadamard spaces, ie complete simply connected metric spaces of nonpositive Alexandrov curvature, and $(X, d)$ the product $X_{1} \times X_{2}$ endowed with the metric $d=\sqrt{d_{1}^{2}+d_{2}^{2}}$. Assume moreover that $X_{1}, X_{2}$ are locally compact. Each metric space $X, X_{1}, X_{2}$ can be compactified by adding its geometric boundary $\partial X, \partial X_{1}, \partial X_{2}$ endowed with the cone topology (see Ballmann [2, Chapter II]). It is well-known that the regular geometric boundary $\partial X^{\text {reg }}$ of $X$ - which consists of the set of equivalence classes of geodesic rays which do not project to a point in one of the factors - is a dense open subset of $\partial X$ homeomorphic to $\partial X_{1} \times \partial X_{2} \times(0, \pi / 2)$. The last factor in this product is called the slope of a point in $\partial X^{\mathrm{reg}}$. The singular geometric boundary $\partial X^{\text {sing }}=\partial X \backslash \partial X^{\text {reg }}$ consists of two strata homeomorphic to $\partial X_{1}$, $\partial X_{2}$ respectively. We assign slope 0 to the first and slope $\pi / 2$ to the second one. 
For a group $\Gamma \subseteq \operatorname{Is}\left(X_{1}\right) \times \operatorname{Is}\left(X_{2}\right)$ acting properly discontinuously by isometries on $X$ we study the limit set $L_{\Gamma}:=\overline{\Gamma \cdot x} \cap \partial X$, where $x \in X$ is arbitrary. Unlike in the case of CAT $(-1)$-spaces, this geometric limit set is not necessarily a minimal set for the action of $\Gamma$ because an element of $\operatorname{Is}\left(X_{1}\right) \times \operatorname{Is}\left(X_{2}\right)$ cannot change the slope $\theta$ of a point in $\partial X$. This is similar to the situation in symmetric spaces or Bruhat-Tits buildings of higher rank. So by abuse of notation we are going to call the set $\partial^{F} X:=\partial X_{1} \times \partial X_{2}$ the Furstenberg boundary, and the projection of $L_{\Gamma} \cap \partial X^{\mathrm{reg}}$ to $\partial X_{1} \times \partial X_{2}$ the Furstenberg limit set $F_{\Gamma}$ of $\Gamma$.

In this note we restrict our attention to discrete groups $\Gamma \subseteq \operatorname{Is}\left(X_{1}\right) \times \operatorname{Is}\left(X_{2}\right)$ which contain an element projecting to a rank one element in each factor, ie $\Gamma$ contains an element $h=\left(h_{1}, h_{2}\right)$ such that the invariant geodesics of $h_{1}, h_{2}$ do not bound a flat half-plane in $X_{1}, X_{2}$. Such an isometry of $X$ will be called regular axial in the sequel. Moreover, for Theorems A and B below we require as in Dal'Bo and Kim [13] that for $i=1,2$ the projection $\Gamma_{i}$ of $\Gamma$ to $\operatorname{Is}\left(X_{i}\right)$ is strongly nonelementary: This means that $\Gamma_{i}, i=1,2$, possesses infinitely many limit points and does not globally fix a point at infinity. By Proposition 3.4 in Caprace and Fujiwara [10], this condition is equivalent to the fact that both $\Gamma_{1}$ and $\Gamma_{2}$ contain a pair of independent rank one elements. For Theorems C, D and E below we need a slightly stronger assumption: We require that $\Gamma \subseteq \operatorname{Is}\left(X_{1}\right) \times \operatorname{Is}\left(X_{2}\right)$ contains two regular axial isometries $g=\left(g_{1}, g_{2}\right)$ and $h=\left(h_{1}, h_{2}\right)$ such that $g_{1}, h_{1}$ and $g_{2}, h_{2}$ are pairs of independent rank one elements in $\operatorname{Is}\left(X_{1}\right)$, resp. Is $\left(X_{2}\right)$.

One important class of examples satisfying our stronger assumption are Kac-Moody groups $\Gamma$ over a finite field which act by isometries on a product $X=X_{1} \times X_{2}$, the CAT(0)-realization of the associated twin building $\mathcal{B}_{+} \times \mathcal{B}_{-}$. Indeed, there exists an element $h=\left(h_{1}, h_{2}\right)$ projecting to a rank one element in each factor by Remark 5.4 and the proof of Corollary 1.3 in [10]. Moreover, the action of the Weyl group produces many regular axial isometries $g=\left(g_{1}, g_{2}\right)$ with $g_{i}$ independent from $h_{i}$ for $i=1,2$. Notice that if the order of the ground field is sufficiently large, then $\Gamma \subseteq \operatorname{Is}\left(X_{1}\right) \times \operatorname{Is}\left(X_{2}\right)$ is an irreducible lattice (see eg Rémy [18] and Caprace and Rémy [11]).

A second type of examples are groups acting properly discontinuously on a product of locally compact Hadamard spaces of strictly negative Alexandrov curvature (compare Dal'Bo and Kim [13] in the manifold setting). In this special case every nonelliptic and nonparabolic isometry in one of the factors is already a rank one element. Prominent examples here which are already covered by the results of Benoist and Quint are Hilbert modular groups acting as irreducible lattices on a product of hyperbolic planes and graphs of convex cocompact groups of rank one symmetric spaces (see also Burger [8]). But our context is much more general and possible factors include locally finite, not 
necessarily regular trees and Riemannian universal covers of geometric rank one manifolds.

Our first result is:

Theorem A The Furstenberg limit set is minimal, ie $F_{\Gamma}$ is the smallest nonempty, $\Gamma$-invariant closed subset of $\partial^{F} X$.

Moreover we have - as in the case of symmetric spaces or Bruhat-Tits buildings of higher rank - the following structure theorem.

Theorem $\mathbf{B}$ The regular geometric limit set splits as a product $F_{\Gamma} \times P_{\Gamma}$, where $P_{\Gamma} \subseteq(0, \pi / 2)$ denotes the set of slopes of regular limit points.

From here on we will assume that $\Gamma$ contains two regular axial isometries projecting to independent rank one elements in each factor. Let $g_{1} \in \operatorname{Is}\left(X_{1}\right), g_{2} \in \operatorname{Is}\left(X_{2}\right)$ be rank one elements. For $i=1,2$ we denote $g_{i}^{+}$the attractive, $g_{i}^{-}$the repulsive fixed point, and $l_{i}\left(g_{i}\right)$ the translation length, ie the minimum of the set $\left\{d_{i}\left(x_{i}, g_{i} x_{i}\right): x_{i} \in X_{i}\right\}$. If $g=\left(g_{1}, g_{2}\right)$, we put $g^{+}:=\left(g_{1}^{+}, g_{2}^{+}\right), g^{-}:=\left(g_{1}^{-}, g_{2}^{-}\right) \in \partial^{F} X$. Then we have the following two statements:

Theorem C The regular geometric limit set $P_{\Gamma}$ is an interval and we have

$$
P_{\Gamma}=\overline{\left\{\arctan \left(l_{2}\left(g_{2}\right) / l_{1}\left(g_{1}\right)\right):\left(g_{1}, g_{2}\right) \in \Gamma, g_{1}, g_{2} \text { rank one }\right\}} \cap(0, \pi / 2) .
$$

Theorem D The set of pairs of fixed points $\left(g^{+}, g^{-}\right) \subset \partial^{F} X \times \partial^{F} X$ of regular axial isometries in $\Gamma$ is dense in $\left(F_{\Gamma} \times F_{\Gamma}\right) \backslash \Delta$, where $\Delta$ denotes the set of points $(\xi, \eta)$ such that $\xi_{1}=\eta_{1}$ or $\xi_{2}=\eta_{2}$.

Notice that Theorem D can be viewed as a strong topological version of the double ergodicity property of Poisson boundaries due to Burger and Monod [9] and Kaimanovich [14].

We next fix a base point $o \in X, \theta \in[0, \pi / 2]$ and consider the cardinality of the sets

$$
N_{\theta}^{\varepsilon}(n):=\left\{\gamma \in \Gamma: n-1<d(o, \gamma o) \leq n,\left|\frac{d_{2}\left(p_{2}(\gamma o), p_{2}(o)\right)}{d_{1}\left(p_{1}(\gamma o), p_{1}(o)\right)}-\tan \theta\right|<\varepsilon\right\},
$$

where $\varepsilon>0$ and $n \in \mathbb{N}$ is large. This number counts orbit points with a correlation of distances to the origin in each factor given by approximately $\tan \theta$. We further define

$$
\delta_{\theta}^{\varepsilon}:=\limsup _{n \rightarrow \infty} \frac{\log N_{\theta}^{\varepsilon}(n)}{n} \text { and } \delta_{\theta}(\Gamma):=\liminf _{\varepsilon \rightarrow 0} \delta_{\theta}^{\varepsilon} .
$$


The quantity $\delta_{\theta}(\Gamma)$ can be thought of as a function of $\theta \in[0, \pi / 2]$ which describes the exponential growth rate of orbit points converging to limit points of slope $\theta$. It is an invariant of $\Gamma$ which carries more information than the critical exponent $\delta(\Gamma)$ : the critical exponent is simply the maximum of $\delta_{\theta}(\Gamma)$ in $[0, \pi / 2]$. As in Quint [16] it will be convenient to study the homogeneous function

$$
\Psi_{\Gamma}: \mathbb{R}_{\geq 0}^{2} \rightarrow \mathbb{R}, H=\left(H_{1}, H_{2}\right) \mapsto\|H\| \cdot \delta_{\arctan \left(H_{1} / H_{2}\right)}(\Gamma) .
$$

Similar to the case of symmetric spaces or Euclidean buildings of higher rank, we have the following:

Theorem $\mathbf{E} \quad \Psi_{\Gamma}$ is upper semicontinuous and concave.

One of the main applications of Theorem $\mathrm{E}$ is that it allows to construct generalized conformal densities on each $\Gamma$-invariant subset of the limit set as in Link [15] and Quint [17] for higher rank symmetric spaces and Euclidean buildings. In a future work we will carry out this construction and relate $\delta_{\theta}(\Gamma)$ to the Hausdorff dimension of the limit set.

The paper is organized as follows: Section 2 recalls basic facts about Hadamard spaces and rank one isometries. In Section 3 we collect properties of products of Hadamard spaces. In Section 4 we study the structure of the limit set and prove Theorems A and B. Section 5 deals with properties of the set of regular axial isometries and contains the proofs of Theorems C and D. In Section 6 we introduce and study the exponent of growth of slope $\theta$ for $\Gamma$. Finally, in Section 7 we construct a so-called generic product for $\Gamma$ in order to show that the function $\Psi_{\Gamma}$ is concave, and give the proof of Theorem E.

Acknowledgements This paper was written during the author's stay at ETH Zurich which was partially supported by the FNS grant PP002-102765. She warmly thanks Marc Burger and Alessandra Iozzi for inviting her, and the FIM for its hospitality and the inspiring atmosphere. She is grateful to Pierre-Emmanuel Caprace for many helpful remarks and discussions and to Françoise Dal'Bo for her valuable comments on a first draft of the paper. She also thanks both referees for their useful suggestions and in particular a considerable simplification of the proof of Lemma 3.1.

\section{Preliminaries}

The purpose of this section is to introduce some terminology and notation and to summarize basic results about Hadamard spaces and rank one isometries. The main 
references here are Bridson and Haefliger [7] and Ballmann [2] (see also Ballmann and Brin [3] and Ballmann, Gromov and Schroeder [4] and Ballmann [1] in the case of Hadamard manifolds).

Let $(X, d)$ be a metric space. A geodesic path joining $x \in X$ to $y \in X$ is a map $\sigma$ from a closed interval $[0, l] \subset \mathbb{R}$ to $X$ such that $\sigma(0)=x, \sigma(l)=y$ and $d\left(\sigma(t), \sigma\left(t^{\prime}\right)\right)=\left|t-t^{\prime}\right|$ for all $t, t^{\prime} \in[0, l]$. We will denote such a geodesic path $\sigma_{x, y} . X$ is called geodesic, if any two points in $X$ can be connected by a geodesic path, if this path is unique, we say that $X$ is uniquely geodesic. In this text $X$ will be a Hadamard space, ie a complete geodesic metric space in which all triangles satisfy the CAT(0)-inequality. This implies in particular that $X$ is simply connected and uniquely geodesic. A geodesic or geodesic line in $X$ is a map $\sigma: \mathbb{R} \rightarrow X$ such that $d\left(\sigma(t), \sigma\left(t^{\prime}\right)\right)=\left|t-t^{\prime}\right|$ for all $t, t^{\prime} \in \mathbb{R}$, a geodesic ray is a map $\sigma:[0, \infty) \rightarrow X$ such that $d\left(\sigma(t), \sigma\left(t^{\prime}\right)\right)=\left|t-t^{\prime}\right|$ for all $t, t^{\prime} \in[0, \infty)$. Notice that in the non-Riemannian setting completeness of $X$ does not imply that every geodesic path or ray can be extended to a geodesic, ie $X$ need not be geodesically complete.

From here on we will assume that $X$ is a locally compact Hadamard space. The geometric boundary $\partial X$ of $X$ is the set of equivalence classes of asymptotic geodesic rays endowed with the cone topology (see eg Ballmann [2, Chapter II]). The action of the isometry group $\operatorname{Is}(X)$ on $X$ naturally extends to an action by homeomorphisms on the geometric boundary. Moreover, since $X$ is locally compact, this boundary $\partial X$ is compact and the space $X$ is a dense and open subset of the compact space $\bar{X}:=X \cup \partial X$. For $x \in X$ and $\xi \in \partial X$ arbitrary, there exists a geodesic ray emanating from $x$ which belongs to the class of $\xi$. We will denote such a ray $\sigma_{x, \xi}$.

We say that two points $\xi, \eta \in \partial X$ can be joined by a geodesic if there exists a geodesic $\sigma: \mathbb{R} \rightarrow X$ such that $\sigma(-\infty)=\xi$ and $\sigma(\infty)=\eta$. It is well-known that if $X$ is CAT $(-1)$, ie of negative Alexandrov curvature bounded above by -1 , then every pair of distinct points in the geometric boundary can be joined by a geodesic. This is not true in general. For convenience we therefore define the visibility set at infinity $\operatorname{Vis}^{\infty}(\xi)$ of a point $\xi \in \partial X$ as the set of points in the geometric boundary which can be joined to $\xi$ by a geodesic, ie

$$
\operatorname{Vis}^{\infty}(\xi):=\{\eta \in \partial X \mid \exists \text { geodesic } \sigma \text { such that } \sigma(-\infty)=\xi, \sigma(\infty)=\eta\}
$$

Let $x, y \in X, \xi \in \partial X$ and $\sigma$ a geodesic ray in the class of $\xi$. We put

$$
\mathcal{B}_{\xi}(x, y):=\lim _{s \rightarrow \infty}(d(x, \sigma(s))-d(y, \sigma(s))) .
$$


This number is independent of the chosen ray $\sigma$, and the function

$$
\begin{aligned}
\mathcal{B}_{\xi}(\cdot, y): X & \rightarrow \mathbb{R} \\
x & \mapsto \mathcal{B}_{\xi}(x, y)
\end{aligned}
$$

is called the Busemann function centered at $\xi$ based at $y$ (see also [2, Chapter II]). For any $x, y, z \in X, \xi \in \partial X$ and $g \in \operatorname{Is}(X)$ the Busemann function satisfies

$$
\begin{aligned}
\left|\mathcal{B}_{\xi}(x, y)\right| & \leq d(x, y) \\
\mathcal{B}_{\xi}(x, z) & =\mathcal{B}_{\xi}(x, y)+\mathcal{B}_{\xi}(y, z) \\
\mathcal{B}_{g \cdot \xi}(g \cdot x, g \cdot y) & =\mathcal{B}_{\xi}(x, y) .
\end{aligned}
$$

A geodesic $\sigma: \mathbb{R} \rightarrow X$ is said to bound a flat half-plane if there exists a closed convex subset $i([0, \infty) \times \mathbb{R})$ in $X$ isometric to $[0, \infty) \times \mathbb{R}$ such that $\sigma(t)=i(0, t)$ for all $t \in \mathbb{R}$. Similarly, a geodesic $\sigma: \mathbb{R} \rightarrow X$ bounds a flat strip of width $c>0$ if there exists a closed convex subset $i([0, c] \times \mathbb{R})$ in $X$ isometric to $[0, c] \times \mathbb{R}$ such that $\sigma(t)=i(0, t)$ for all $t \in \mathbb{R}$. We call a geodesic $\sigma: \mathbb{R} \rightarrow X$ a rank one geodesic if $\sigma$ does not bound a flat half-plane.

The following important lemma states that even though we cannot join any two distinct points in the geometric boundary of $X$, given a rank one geodesic we can at least join points in a neighborhood of its extremities. More precisely, we have the following well-known lemma:

Lemma 2.1 [2, Lemma III.3.1] Let $\sigma: \mathbb{R} \rightarrow X$ be a rank one geodesic which does not bound a flat strip of width $c$. Then there are neighborhoods $U$ of $\sigma(-\infty)$ and $V$ of $\sigma(\infty)$ in $\bar{X}$ such that for any $\xi \in U$ and $\eta \in V$ there exists a rank one geodesic joining $\xi$ and $\eta$. For any such geodesic $\sigma^{\prime}$ we have $d\left(\sigma^{\prime}, \sigma(0)\right) \leq c$.

Moreover, we will need the following technical lemma which immediately follows from Lemmas 4.3 and 4.4 in [3].

Lemma 2.2 Let $\sigma: \mathbb{R} \rightarrow X$ be a rank one geodesic and put $y:=\sigma(0), \eta:=\sigma(\infty)$. Then for any $T \gg 1, \varepsilon>0$ there exists a neighborhood $U$ of $\sigma(-\infty)$ in $\bar{X}$ and a number $R>0$ such that for any $x \in X$ with $d(x, \sigma)>R$ or $x \in U$ we have

$$
d\left(\sigma_{x, y}(t), \sigma_{x, \eta}(t)\right) \leq \varepsilon \quad \text { for all } t \in[0, T]
$$


The following kind of isometries will play a central role in the sequel.

Definition 2.3 An isometry $h$ of $X$ is called axial, if there exists a constant $l=l(h)>0$ and a geodesic $\sigma$ such that $h(\sigma(t))=\sigma(t+l)$ for all $t \in \mathbb{R}$. We call $l(h)$ the translation length of $h$, and $\sigma$ an axis of $h$. The boundary point $h^{+}:=\sigma(\infty)$ is called the attractive fixed point, and $h^{-}:=\sigma(-\infty)$ the repulsive fixed point of $h$. We further put $\operatorname{Ax}(h):=\{x \in X \mid d(x, h x)=l(h)\}$.

We remark that $\operatorname{Ax}(h)$ consists of the union of parallel geodesics translated by $h$, and $\overline{\mathrm{Ax}(h)} \cap \partial X$ is exactly the set of fixed points of $h$. Moreover, we have the following easy formula for the translation length of an axial isometry in terms of Busemann functions.

Lemma 2.4 If $h$ is an axial isometry with attractive and repulsive fixed points $h^{+}$, $h^{-}$then its translation length is given by

$$
l(h)=\mathcal{B}_{h^{+}}(x, h x)=\mathcal{B}_{h^{-}}(h x, x), \quad \text { where } x \in X \text { is arbitrary } .
$$

Proof Let $x, y \in X$ arbitrary. Then by the cocycle identity (4) and the fact that $h$ fixes $h^{+}$and $h^{-}$,

$$
\begin{aligned}
\mathcal{B}_{h^{+}}(x, h x) & =\mathcal{B}_{h^{+}}(x, y)+\mathcal{B}_{h^{+}}(y, h y)+\mathcal{B}_{h^{+}}(h y, h x) \\
& =\mathcal{B}_{h^{+}}(x, y)+\mathcal{B}_{h^{+}}(y, h y)+\underbrace{\mathcal{B}_{h^{+}}(y, x)}_{=-\mathcal{B}_{h^{+}}(x, y)}=\mathcal{B}_{h^{+}}(y, h y),
\end{aligned}
$$

and similarly $\mathcal{B}_{h^{-}}(h x, x)=\mathcal{B}_{h^{-}}(h y, y)$. So the terms on the right-hand side are independent of $x \in X$, and choosing $x \in \operatorname{Ax}(h)$ yields the claim.

Following the definition in $[6 ; 10]$ we will call two axial isometries $g, h \in \operatorname{Is}(X)$ independent if for any given $x \in X$ the map

$$
\mathbb{Z} \times \mathbb{Z} \rightarrow[0, \infty),(m, n) \mapsto d\left(g^{m} x, h^{m} x\right)
$$

is proper.

Definition 2.5 An axial isometry is called rank one if it possesses a rank one axis.

Notice that if $h$ is rank one, then $h^{+}$and $h^{-}$are the only fixed points of $h$. Moreover, it is easy to verify that two rank one elements $g, h \in \operatorname{Is}(X)$ are independent if and only if $\left\{g^{+}, g^{-}\right\} \cap\left\{h^{+}, h^{-}\right\}=\varnothing$. Let us recall some properties of rank one isometries. 
Lemma 2.6 [2, Lemma III.3.3] Let $h$ be a rank one isometry. Then

(a) $\operatorname{Vis}^{\infty}\left(h^{+}\right)=\partial X \backslash\left\{h^{+}\right\}$,

(b) any geodesic joining a point $\xi \in \partial X \backslash\left\{h^{+}\right\}$to $h^{+}$is rank one,

(c) given neighborhoods $U$ of $h^{-}$and $V$ of $h^{+}$in $\bar{X}$ there exists $N_{0} \in \mathbb{N}$ such that $h^{-n}(\bar{X} \backslash V) \subset U$ and $\quad h^{n}(\bar{X} \backslash U) \subset V$ for all $n \geq N_{0}$.

In particular, by (c) we have $\lim _{n \rightarrow \infty} h^{-n} \xi=h^{-}$for any $\xi \in \operatorname{Vis}^{\infty}\left(h^{+}\right)$. The following lemma will be central for the proof of Theorem 5.2.

Lemma 2.7 [2, Lemma III.3.2] Let $\sigma: \mathbb{R} \rightarrow X$ be a rank one geodesic, and $\left(\gamma_{n}\right) \subset$ $\operatorname{Is}(X)$ a sequence of isometries such that $\gamma_{n} x \rightarrow \sigma(\infty)$ and $\gamma_{n}^{-1} x \rightarrow \sigma(-\infty)$ for one (and hence any) $x \in X$. Then for $n$ sufficiently large, $\gamma_{n}$ is axial and possesses an axis $\sigma_{n}$ such that $\sigma_{n}(\infty) \rightarrow \sigma(\infty)$ and $\sigma_{n}(-\infty) \rightarrow \sigma(-\infty)$.

The following proposition is a generalization of Lemma 4.1 in [12]. It gives a relation between the geometric length and the combinatorial length of words in a free group on two generators which will be a clue to the proof of Theorem 5.2. Our proof here involves a new idea since F Dal'Bo's proof is based on the fact that $X$ is CAT(-1) and hence triangles in $X$ are thinner than the corresponding triangles in hyperbolic space. If $g, h$ generate a free group we say that a word $\gamma=s_{1}^{k_{1}} s_{2}^{k_{2}} \cdots s_{n}^{k_{n}}$ with $s_{i} \in\left\{g, g^{-1}, h, h^{-1}\right\}$ and $k_{i} \in \mathbb{N} \backslash\{0\}, i \in\{1,2, \ldots, n\}$ is cyclically reduced if $s_{i+1} \notin\left\{s_{i}, s_{i}^{-1}\right\}, i \in\{1,2, \ldots, n-1\}$, and $s_{n} \neq s_{1}^{-1}$.

Proposition 2.8 Suppose $g$ and $h$ are rank one elements in $\operatorname{Is}(X)$ with pairwise distinct fixed points. Then there exists $N \in \mathbb{N}$ and $C>0$ such that for all $n \in \mathbb{N}$ and any cyclically reduced word $\gamma=s_{1}^{k_{1}} s_{2}^{k_{2}} \cdots s_{n}^{k_{n}}$ with $s_{i} \in S:=\left\{g^{N}, g^{-N}, h^{N}, h^{-N}\right\}$ and $k_{i} \in \mathbb{N} \backslash\{0\}, i \in\{1,2, \ldots, n\}$, we have

$$
\left|l(\gamma)-\sum_{i=1}^{n} k_{i} l\left(s_{i}\right)\right| \leq C \cdot n .
$$

Proof We fix some base point $o \in X$. For $\eta \in\left\{g^{-}, g^{+}, h^{-}, h^{+}\right\}$let $U(\eta) \subset \bar{X}$ be a small neighborhood of $\eta$ with $o \notin U(\eta)$ such that all $U(\eta)$ are pairwise disjoint, and $c>0$ a constant such that any pair of points in distinct neighborhoods can be joined by a rank one geodesic $\sigma^{\prime}$ with $d\left(o, \sigma^{\prime}\right) \leq c$. This is possible by Lemma 2.1. According to Lemma 2.7 there exist neighborhoods $W(\eta) \subseteq U(\eta), \eta \in\left\{g^{-}, g^{+}, h^{-}, h^{+}\right\}$, such that every $\gamma \in \Gamma$ with $\gamma o \in W(\eta), \gamma^{-1} o \in W(\zeta), \zeta \neq \eta$, is rank one with $\gamma^{+} \in U(\eta)$ 
and $\gamma^{-} \in U(\zeta)$. Moreover, by Lemma 2.6 (c) there exists $N \in \mathbb{N}$ such that for all $\gamma \in\left\{g, g^{-1}, h, h^{-1}\right\}$,

$$
\gamma^{N}\left(\bar{X} \backslash W\left(\gamma^{-}\right)\right) \subseteq W\left(\gamma^{+}\right) .
$$

We put $S:=\left\{g^{N}, g^{-N}, h^{N}, h^{-N}\right\}$ and consider a cyclically reduced word $\gamma=$ $s_{1}^{k_{1}} s_{2}^{k_{2}} \cdots s_{n}^{k_{n}}$ with $s_{i} \in S$ and $k_{i} \in \mathbb{N} \backslash\{0\}, i \in\{1,2, \ldots, n\}$. By the choice of $N$ and (5) we have $\gamma o \in W\left(s_{1}^{+}\right)$and $\gamma^{-1} o \in W\left(s_{n}^{-}\right) \neq W\left(s_{1}^{+}\right)$since $s_{1} \neq s_{n}^{-1}$. Therefore $\gamma$ is rank one with $\gamma^{+} \in U\left(s_{1}^{+}\right)$and $\gamma^{-} \in U\left(s_{n}^{-}\right)$. Choosing a point $x \in \operatorname{Ax}(\gamma)$ with $d(o, x) \leq c$ we get

$$
l(\gamma) \leq d(o, \gamma o) \leq d(o, x)+d(x, \gamma x)+d(\gamma x, \gamma o) \leq l(\gamma)+2 c .
$$

Similarly $l\left(s_{i}^{k_{i}}\right) \leq d\left(o, s_{i}^{k_{i}} o\right) \leq l\left(s_{i}^{k_{i}}\right)+2 c$ for $i \in\{1,2, \ldots, n\}$.

For $i \in\{1,2, \ldots, n\}$ we abbreviate $\gamma_{i}:=s_{i}^{k_{i}} s_{i+1}^{k_{i}+1} \cdots s_{n}^{k_{n}}$. Then $\gamma_{2} o \in W\left(s_{2}^{+}\right)$, $s_{1}^{-k_{1}} o \in W\left(s_{1}^{-}\right) \neq W\left(s_{2}^{+}\right)$, so there exists a geodesic $\sigma_{2}$ joining $\gamma_{2} o$ to $s_{1}^{-k_{1}} o$ with $d\left(o, \sigma_{2}\right) \leq c$. If $y$ denotes a point on $\sigma_{2}$ with $d(o, y) \leq c$ we obtain

$$
d\left(s_{1}^{k_{1}} s_{2}^{k_{2}} \cdots s_{n}^{k_{n}} o, o\right)=d\left(\gamma_{2} o, s_{1}^{-k_{1}} o\right) \leq d\left(\gamma_{2} o, y\right)+d\left(y, s_{1}^{-k_{1}} o\right)
$$

which proves $\left|d(\gamma o, o)-d\left(o, s_{1}^{k_{1}} o\right)-d\left(o, \gamma_{2} o\right)\right| \leq 2 c$. Applying the same arguments to $\gamma_{i}$ for $i \geq 2$ and using $s_{i+1} \neq s_{i}^{-1}$ we deduce $\left|d\left(\gamma_{i} o, o\right)-d\left(o, s_{i}^{k_{i}} o\right)-d\left(o, \gamma_{i+1} o\right)\right| \leq 2 c$. Therefore

$$
\left|d(o, \gamma o)-\sum_{i=1}^{n} d\left(o, s_{i}^{k_{i}} o\right)\right| \leq 2(n-1) c
$$

and, by (6), $\left|l(\gamma)-\sum_{i=1}^{n} k_{i} l\left(s_{i}\right)\right| \leq 4 c \cdot n$. It remains to set $C:=4 c$.

Moreover, the following generalization of Lemma 1.4 (2) in [13] will also be needed in the proof of Theorem 5.2:

Lemma 2.9 Suppose $g$ and $h$ are rank one elements in Is $(X)$ with $g^{+}=h^{+}$. Then there exists $N \in \mathbb{N}$ such that for all $n, m \in \mathbb{N} \backslash\{0\}$ the isometry $g^{N n} h^{N m}$ is rank one and

$$
l\left(h^{N n} g^{N m}\right)=N n l(h)+N m l(g) .
$$

Proof As in the proof of the previous proposition we fix some base point $o \in X$ and let $U(\eta) \subset \bar{X}$ be a small neighborhood of $\eta \in\left\{g^{-}, g^{+}, h^{-}\right\}$with $o \notin U(\eta)$ such that all $U(\eta)$ are pairwise disjoint. Notice that by our assumption we may set $U\left(h^{+}\right):=U\left(g^{+}\right)$. Fix neighborhoods $W(\eta) \subseteq U(\eta), \eta \in\left\{g^{-}, g^{+}, h^{-}, h^{+}\right\}$, such that every $\gamma \in \Gamma$ with 
$\gamma o \in W(\eta), \gamma^{-1} o \in W(\zeta), \zeta \neq \eta$, is rank one with $\gamma^{+} \in U(\eta)$ and $\gamma^{-} \in U(\zeta)$, and $N \in \mathbb{N}$ such that for all $\gamma \in\left\{g, g^{-1}, h, h^{-1}\right\}$,

$$
\gamma^{N}\left(\bar{X} \backslash W\left(\gamma^{-}\right)\right) \subseteq W\left(\gamma^{+}\right) .
$$

Then for $n, m \in \mathbb{N} \backslash\{0\} h^{N n} g^{N m} o \in W\left(h^{+}\right)$and $\left(h^{N n} g^{N m}\right)^{-1} o \in W\left(g^{-}\right) \neq W\left(h^{+}\right)$, hence $\gamma:=h^{N n} g^{N m}$ is rank one with $\gamma^{+} \in U\left(h^{+}\right)$and $\gamma^{-} \in U\left(g^{-}\right)$. Furthermore, $\gamma h^{+}=h^{N n} g^{N m} h^{+}=h^{+}$implies that $h^{+}$is one of the two fixed points of $\gamma$, hence $\gamma^{+}=h^{+}=g^{+}$. We conclude using Lemma 2.4 and the cocycle identity (4) that

$$
\begin{aligned}
l(\gamma)=\mathcal{B}_{\gamma^{+}}(o, \gamma o) & =\mathcal{B}_{h^{+}}\left(o, h^{N n} o\right)+\mathcal{B}_{h^{+}}\left(h^{N n} o, h^{N n} g^{N m} o\right) \\
& =l\left(h^{N n}\right)+\mathcal{B}_{g^{+}}\left(o, g^{N m} o\right)=N n l(h)+N m l(g) .
\end{aligned}
$$

If $\Gamma$ is a group acting by isometries on a locally compact Hadamard space $X$ we define its geometric limit set by $L_{\Gamma}:=\overline{\Gamma \cdot x} \cap \partial X$, where $x \in X$ is arbitrary.

From here on we let $\Gamma \subset \operatorname{Is}(X)$ be a (not necessarily discrete) group which possesses a rank one element $h$. Denote $\sigma$ an axis of $h$ and put $o:=\sigma(0)$. The idea of proof of the following three facts is due to W Ballmann (see eg the proof of Theorem 2.8 in [1]). We include complete proofs for the convenience of the reader.

Lemma 2.10 If $\Gamma$ does not globally fix a point in $\partial X$, then for any neighborhood $V$ of $\xi \in L_{\Gamma}$ in $\bar{X}$ there exists $\gamma \in \Gamma$ such that $\gamma h^{+} \in V$.

Proof Choose $\left(\gamma_{n}\right) \subset \Gamma$ such that $\gamma_{n} o \rightarrow \xi$ as $n \rightarrow \infty$. Passing to a subsequence if necessary we may assume that $\gamma_{n}^{-1} o$ converges to a point $\zeta \in L_{\Gamma}$ as $n \rightarrow \infty$. Let $T \gg 1$ and $\varepsilon>0$ be arbitrary. By Lemma 2.2 there exist a constant $R>0$ and a neighborhood $U$ of $h^{-}=\sigma(-\infty)$ in $\bar{X}$ such that for any $x \in X$ with $d(x, \sigma)>R$ or $x \in U$ we have $d\left(\sigma_{x, o}(t), \sigma_{x, h^{+}}(t)\right) \leq \varepsilon / 2$ for all $t \in[0, T]$.

We first treat the case $\zeta \notin\left\{h^{+}, h^{-}\right\}$. Then for $n$ sufficiently large we have $d\left(\gamma_{n}^{-1} o, \sigma\right)>$ $R$ and $d\left(\sigma_{o, \gamma_{n} o}(t), \sigma_{o, \xi}(t)\right) \leq \varepsilon / 2$ for $0 \leq t \leq T$. We conclude that for $t \in[0, T]$,

$$
\begin{aligned}
d\left(\sigma_{o, \xi}(t), \sigma_{o, \gamma_{n} h^{+}}(t)\right) & \leq d\left(\sigma_{o, \xi}(t), \sigma_{o, \gamma_{n} o}(t)\right)+d\left(\sigma_{o, \gamma_{n} o}(t), \sigma_{o, \gamma_{n} h^{+}}(t)\right) \\
& \leq \frac{\varepsilon}{2}+d\left(\gamma_{n} \sigma_{\gamma_{n}^{-1} o, o}(t), \gamma_{n} \sigma_{\gamma_{n}^{-1} o, h^{+}}(t)\right) \\
& =\frac{\varepsilon}{2}+\underbrace{d\left(\sigma_{\gamma_{n}^{-1} o, o}(t), \sigma_{\gamma_{n}^{-1} o, h^{+}}(t)\right)}_{\leq \varepsilon / 2} \leq \varepsilon
\end{aligned}
$$

which proves the assertion in this case.

If $\zeta=h^{-}$then $\gamma_{n}^{-1} o \in U$ for $n$ sufficiently large, hence by Lemma 2.2 and the above inequalities the claim also holds. 
Now assume that $\zeta=h^{+}$. Since $\Gamma$ does not globally fix a point in $\partial X$ there exists $\varphi \in \Gamma$ such that $\varphi \zeta \neq h^{+}$. Then, replacing $\gamma_{n}$ by $\gamma_{n} \varphi^{-1}$ and using the fact that $\gamma_{n} \varphi^{-1} o \rightarrow \xi$ and $\varphi \gamma_{n}^{-1} o \rightarrow \varphi \zeta \neq h^{+}$, we are in one of the cases above. Hence the assertion follows.

The following result will be one of the key lemmas for the product case in Section 4 .

Lemma 2.11 If $\Gamma$ does not globally fix a point in $\partial X$ and $\# L_{\Gamma}=\infty$, then for all $\xi$, $\eta, \zeta \in L_{\Gamma}$ there exists $\gamma \in \Gamma$ such that $\gamma \xi \neq \zeta$ and $\gamma \xi \neq \eta$.

Proof If $\xi \in L_{\Gamma} \backslash\{\zeta, \eta\}$ we can take $\gamma=e$ (the identity in $\Gamma$ ).

Suppose now $\xi=\zeta \neq \eta$ and $\xi \notin\left\{h^{+}, h^{-}\right\}$. Then $h^{n} \xi \rightarrow h^{+}$as $n \rightarrow \infty$. If $\eta \neq h^{+}$, let $V$ be a neighborhood of $h^{+}$disjoint from $\xi, \eta$. Then there exists $N \in \mathbb{N}$ such that $h^{n} \xi \in V$ for all $n \geq N$, in particular $h^{N} \xi \neq \xi=\zeta$ and $h^{N} \xi \neq \eta$. If $\eta=h^{+}$we choose a neighborhood $V$ of $h^{+}$disjoint from $\xi$ and let $N \in \mathbb{N}$ such that $h^{n} \xi \in V$ for all $n \geq N$. If $h^{n} \xi=\eta=h^{+}$for all $n \geq N$, then $\xi$ is a fixed point of $h$ which is a contradiction to $\xi \notin\left\{h^{+}, h^{-}\right\}$. Hence there exists $n \geq N$ such that $h^{n} \xi \neq \eta$ and $h^{n} \xi \neq \xi=\zeta$.

If $\xi=\zeta=h^{+}, \eta \neq h^{+}$we choose a point in $L_{\Gamma} \backslash\left\{h^{+}, \eta\right\}$ and a neighborhood $V$ of this point disjoint from $\left\{h^{+}, \eta\right\}$. By Lemma 2.10 there exists $\gamma \in \Gamma$ such that $\gamma h^{+} \in V$, in particular $\gamma h^{+} \notin\left\{h^{+}, \eta\right\}$.

Replacing $h$ by $h^{-1}$ in the previous argument yields the assertion for the case $\xi=\zeta=$ $h^{-}, \eta \neq h^{-}$.

By symmetry, the claim also holds for $\xi=\eta \neq \zeta$.

The remaining case is $\xi=\zeta=\eta$. Since $\Gamma$ does not globally fix a point in $\partial X$, there exists $\gamma \in \Gamma$ such that $\gamma \xi \neq \xi$.

In the case of discrete groups, the following result is part of Theorem 2.8 in [1]. Since we are dealing here with possibly nondiscrete groups we have to add the condition that $\Gamma$ does not globally fix a point in $\partial X$. This excludes for example the case of a group consisting of infinitely many rank one elements with a common fixed point at infinity.

Proposition 2.12 If $\Gamma$ does not globally fix a point in $\partial X$ and $\# L_{\Gamma}=\infty$, then the limit set $L_{\Gamma}$ is minimal, ie the smallest nonempty $\Gamma$-invariant closed subset of $\partial X$. 
Proof We first notice that every nonempty $\Gamma$-invariant closed subset $A$ of $\partial X$ contains a limit point: Indeed, if $\xi \in A$, then either $\xi=h^{+}$or $\xi \in \operatorname{Vis}{ }^{\infty}\left(h^{+}\right)$. So either $A$ contains the limit point $h^{+}$or the point $h^{-}=\lim _{n \rightarrow \infty} h^{-n} \xi$.

Next we fix $\xi \in L_{\Gamma}$ and let $\eta \in L_{\Gamma}$ be arbitrary. Our goal is to show that $\eta \in \overline{\Gamma \cdot \xi}$.

Let $U \subset \partial X$ be an arbitrary neighborhood of $\eta$. By Lemma 2.10 there exists $\gamma \in \Gamma$ such that $\gamma h^{+} \in U$. Hence if $\xi \neq \gamma h^{-}$, by the dynamics of rank one isometries Lemma 2.6 (c), $\left(\gamma h \gamma^{-1}\right)^{n} \xi \in U$ for $n$ sufficiently large. If $\xi=\gamma h^{-}$, there exists $\varphi \in \Gamma$ such that $\varphi \xi \neq \gamma h^{-}$by Lemma 2.11. Then $\left(\gamma h \gamma^{-1}\right)^{n} \varphi \xi \in U$ for $n$ sufficiently large. $\square$

\section{Products of Hadamard spaces}

Now let $\left(X_{1}, d_{1}\right),\left(X_{2}, d_{2}\right)$ be locally compact Hadamard spaces, and $X=X_{1} \times X_{2}$ the product space endowed with the product distance $d=\sqrt{d_{1}^{2}+d_{2}^{2}}$. Notice that such a product is again a locally compact Hadamard space. To any pair of points $x=\left(x_{1}, x_{2}\right), z=\left(z_{1}, z_{2}\right) \in X$ we associate the vector

$$
H(x, z):=\left(\begin{array}{l}
d_{1}\left(x_{1}, z_{1}\right) \\
d_{2}\left(x_{2}, z_{2}\right)
\end{array}\right) \in \mathbb{R}^{2},
$$

which we call the distance vector of the pair $(x, z)$. If $z \neq x$ we further define the direction of $z$ with respect to $x$ by

$$
\theta(x, z):=\arctan \frac{d_{2}\left(x_{2}, z_{2}\right)}{d_{1}\left(x_{1}, z_{1}\right)} .
$$

Notice that we have

$$
H(x, z)=d(x, z)\left(\begin{array}{c}
\cos \theta(x, z) \\
\sin \theta(x, z)
\end{array}\right),
$$

in particular $\|H(x, z)\|=d(x, z)$, where $\|\cdot\|$ denotes the Euclidean norm in $\mathbb{R}^{2}$.

Denote $p_{i}: X \rightarrow X_{i}, i=1,2$, the natural projections. Every geodesic path $\sigma:[0, l] \rightarrow$ $X$ can be written as a product $\sigma(t)=\left(\sigma_{1}(t \cos \theta), \sigma_{2}(t \sin \theta)\right)$, where $\theta \in[0, \pi / 2]$ and $\sigma_{1}:[0, l \cos \theta] \rightarrow X_{1}, \sigma_{2}:[0, l \sin \theta] \rightarrow X_{2}$ are geodesic paths in $X_{1}, X_{2} . \theta$ equals the direction of $\sigma(l)$ with respect to $\sigma(0)$ and is called the slope of $\sigma$. We say that a geodesic path $\sigma$ is regular if its slope is contained in the open interval $(0, \pi / 2)$. In other words, $\sigma$ is regular if neither $p_{1}(\sigma([0, l]))$ nor $p_{2}(\sigma([0, l]))$ is a point.

If $x \in X$ and $\sigma:[0, \infty) \rightarrow X$ is an arbitrary geodesic ray, then by elementary geometric estimates one has the relation

$$
\theta=\lim _{t \rightarrow \infty} \theta(x, \sigma(t))
$$


between the slope $\theta$ of $\sigma$ and the directions of $\sigma(t), t>0$, with respect to $x$. Similarly, one can easily show that any two geodesic rays representing the same (possibly singular) point in the geometric boundary necessarily have the same slope. So we may define the slope $\theta(\widetilde{\xi})$ of a point $\tilde{\xi} \in \partial X$ as the slope of an arbitrary geodesic ray representing $\tilde{\xi}$. Moreover, two regular geodesic rays $\sigma, \sigma^{\prime}$ with the same slope represent the same point in the geometric boundary if and only if $\sigma_{1}(\infty)=\sigma_{1}^{\prime}(\infty)$ and $\sigma_{2}(\infty)=\sigma_{2}^{\prime}(\infty)$. The regular geometric boundary $\partial X^{\mathrm{reg}}$ of $X$ is defined as the set of equivalence classes of regular geodesic rays and hence is homeomorphic to $\partial X_{1} \times \partial X_{2} \times(0, \pi / 2)$.

If $\gamma \in \operatorname{Is}\left(X_{1}\right) \times \operatorname{Is}\left(X_{2}\right)$, then the slope of $\gamma \cdot \widetilde{\xi}$ equals the slope of $\widetilde{\xi}$. In other words, if $\partial X_{\theta}$ denotes the set of points in the geometric boundary of slope $\theta \in[0, \pi / 2]$, then $\partial X_{\theta}$ is invariant by the action of $\operatorname{Is}\left(X_{1}\right) \times \operatorname{Is}\left(X_{2}\right)$. Notice that points in $\partial X^{\text {sing }}:=$ $(\partial X)_{0} \cup(\partial X)_{\pi / 2}$ are equivalence classes of geodesic rays which project to a point in one of the factors of $X$. Hence $(\partial X)_{0}$ is homeomorphic to $\partial X_{1}$ and $(\partial X)_{\pi / 2}$ is homeomorphic to $\partial X_{2}$. If $\theta \in(0, \pi / 2)$, then the set $\partial X_{\theta} \subset \partial X^{\text {reg }}$ is homeomorphic to the product $\partial X_{1} \times \partial X_{2}$.

In the case of symmetric spaces and Bruhat-Tits buildings of higher rank there is a well-known notion of Furstenberg boundary, which - for a product of rank one spaces coincides with the product of the geometric boundaries. In our more general setting we therefore choose to call the product $\partial X_{1} \times \partial X_{2}$ endowed with the product topology the Furstenberg boundary $\partial^{F} X$ of $X$. Using the above parametrization of $\partial X^{\text {reg }}$ we have a natural projection

$$
\begin{aligned}
\pi^{F}: \quad \partial X^{\text {reg }} & \rightarrow \partial^{F} X \\
\left(\xi_{1}, \xi_{2}, \theta\right) & \mapsto\left(\xi_{1}, \xi_{2}\right)
\end{aligned}
$$

and a natural action of the group $\operatorname{Is}\left(X_{1}\right) \times \operatorname{Is}\left(X_{2}\right)$ by homeomorphisms on the Furstenberg boundary of $X=X_{1} \times X_{2}$.

We have the following important lemma concerning the topology of $\bar{X}$. Although elementary, we include the proof for the convenience of the reader.

Lemma 3.1 Suppose $\left(y_{n}\right) \subset X$ is a sequence converging to a point $\tilde{\eta} \in \partial X_{\theta}$ for some $\theta \in[0, \pi / 2]$. Then for any $x \in X$ we have $\theta\left(x, y_{n}\right) \rightarrow \theta$ as $n \rightarrow \infty$.

Proof First notice that if $\sigma$ is a geodesic emanating from $x$, then $\theta(x, \sigma(t))$ does not depend on $t$. We define $\sigma$ as a geodesic ray joining $x$ to $\tilde{\eta}$, so in particular $\sigma$ has slope $\theta$ and $\theta(x, \sigma(t))=\theta$ for all $t>0$. Without loss of generality we may assume that $d\left(x, y_{n}\right) \geq 1$ for all $n \in \mathbb{N}$. It therefore remains to prove that $\theta\left(x, \sigma_{x, y_{n}}(1)\right)=\theta\left(x, y_{n}\right)$ converges to $\theta(x, \sigma(1))=\theta$ as $n$ tends to infinity. This is clear since $\sigma_{x, y_{n}}(1)$ converges to $\sigma(1)$ and since the map $z \mapsto \theta(x, z)$ is continuous on every sphere around $x$. 
Recall the definition of visibility set at infinity $\operatorname{Vis}^{\infty}(\widetilde{\xi})$ of a point $\tilde{\xi} \in \partial X$ from (1). It is easy to see that a point $\tilde{\eta} \in \partial X$ cannot belong to $\operatorname{Vis}^{\infty}(\widetilde{\xi})$ if the slope of $\tilde{\eta}$ is different from the slope of $\tilde{\xi}$. This motivates the following less restrictive definition for pairs of points in the Furstenberg boundary: We say that $\xi=\left(\xi_{1}, \xi_{2}\right)$ and $\eta=\left(\eta_{1}, \eta_{2}\right) \in \partial^{F} X$ are opposite if $\xi_{1}$ and $\eta_{1}$ can be joined by a geodesic in $X_{1}$, and $\xi_{2}, \eta_{2}$ can be joined by a geodesic in $X_{2}$. Moreover, the Furstenberg visibility set $\mathrm{Vis}^{F}(\xi)$ of a point $\xi=\left(\xi_{1}, \xi_{2}\right) \in \partial^{F} X$ is defined as the set of points in $\partial^{F} X$ which are opposite to $\xi$, ie

$$
\operatorname{Vis}^{F}(\xi)=\left\{\left(\eta_{1}, \eta_{2}\right) \in \partial^{F} X: \eta_{1} \in \mathrm{Vis}^{\infty}\left(\xi_{1}\right) \text { and } \eta_{2} \in \mathrm{Vis}^{\infty}\left(\xi_{2}\right)\right\} \text {. }
$$

In particular, for any $\tilde{\xi} \in \partial X^{\text {reg }}$ with $\pi^{F}(\tilde{\xi})=\xi$ one has $\operatorname{Vis}^{F}(\xi)=\pi^{F}\left(\operatorname{Vis}^{\infty}(\tilde{\xi})\right)$. So we may alternatively define the Furstenberg visibility set of a point $\xi \in \partial^{F} X$ via

$$
\operatorname{Vis}^{F}(\xi):=\pi^{F}\left(\operatorname{Vis}^{\infty}(\tilde{\xi})\right) \text {, where } \tilde{\xi} \in\left(\pi^{F}\right)^{-1}(\xi) \text { is arbitrary . }
$$

Moreover, in the particular case that both $X_{1}, X_{2}$ are $\operatorname{CAT}(-1)$, for $\widetilde{\xi}=\left(\xi_{1}, \xi_{2}, \theta\right) \in$ $\partial X^{\text {reg }}$ we have

$$
\operatorname{Vis}^{\infty}(\widetilde{\xi})=\left\{\left(\eta_{1}, \eta_{2}, \theta\right) \in \partial X^{\mathrm{reg}}: \eta_{1} \neq \xi_{1} \text { and } \eta_{2} \neq \xi_{2}\right\},
$$

and $\left(\xi_{1}, \xi_{2}\right),\left(\eta_{1}, \eta_{2}\right) \in \partial^{F} X$ are opposite if and only if $\xi_{1} \neq \eta_{1}$ and $\xi_{2} \neq \eta_{2}$.

\section{The structure of the limit set}

Recall that the geometric limit set of a group $\Gamma$ acting by isometries on a locally compact Hadamard space is defined by $L_{\Gamma}:=\overline{\Gamma \cdot x} \cap \partial X$, where $x \in X$ is arbitrary. In this section we will investigate the structure of the geometric limit set of certain groups $\Gamma \subset \operatorname{Is}\left(X_{1}\right) \times \operatorname{Is}\left(X_{2}\right) \subseteq \operatorname{Is}(X)$ acting properly discontinuously on the product $X$ of two locally compact Hadamard spaces $X_{1}, X_{2}$. By abuse of notation we denote $p_{i}: \Gamma \rightarrow \operatorname{Is}\left(X_{i}\right), i=1,2$, the natural projections and put $\Gamma_{i}:=p_{i}(\Gamma), i=1,2$. Notice that $\Gamma_{i}$ need not act properly discontinuously on $X_{i}$. As in [13] for $i \in\{1,2\}$ we call $\Gamma_{i}$ strongly nonelementary if it does not globally fix a point in $\partial X_{i}$ and $\# L_{\Gamma_{i}}$ is infinite.

From here on we assume that $\Gamma \subset \operatorname{Is}\left(X_{1}\right) \times \operatorname{Is}\left(X_{2}\right)$ acts properly discontinuously, the projections $\Gamma_{1}, \Gamma_{2}$ are strongly nonelementary, and $\Gamma$ contains an isometry $h$ such that $h_{1}:=p_{1}(h), h_{2}:=p_{2}(h)$ are rank one elements in $\Gamma_{1}, \Gamma_{2}$ respectively. Such an isometry $h$ will be called regular axial and we will denote $\widetilde{h^{+}}$its attractive fixed point in $\partial X^{\text {reg }}$ and $h^{+}:=\pi^{F}\left(\widetilde{h^{+}}\right)=\left(h_{1}^{+}, h_{2}^{+}\right)$. Notice that (8) and Lemma 2.6 (a) imply

$$
\operatorname{Vis}^{F}\left(h^{+}\right)=\left\{\left(\xi_{1}, \xi_{2}\right) \in \partial^{F} X: \xi_{1} \neq h_{1}^{+}, \xi_{2} \neq h_{2}^{+}\right\} \text {. }
$$

Moreover, by Lemma 2.6 (c) we have $\lim _{n \rightarrow \infty} h^{-n} \xi=h^{-}$for all $\xi \in \operatorname{Vis}^{F}\left(h^{+}\right)$. 
We remark that the existence of a regular axial element in $\Gamma$ imposes severe restrictions on the spaces $X_{1}$ and $X_{2}$. For example, neither $X_{1}$ nor $X_{2}$ can be a higher rank symmetric space or Euclidean building. However, as mentioned in the introduction the buildings associated to Kac-Moody groups over finite fields, Riemannian universal covers of geometric rank one manifolds and CAT $(-1)$-spaces such as locally finite trees or manifolds of pinched negative curvature are natural examples of possible factors.

For convenience we define the Furstenberg limit set of $\Gamma$ by $F_{\Gamma}:=\pi^{F}\left(L_{\Gamma} \cap \partial X^{\mathrm{reg}}\right)$. It is clearly a subset of the product $L_{\Gamma_{1}} \times L_{\Gamma_{2}} \subseteq \partial^{F} X$. Using our Lemma 2.11 the proof of the following important lemma is as for Lemma 2.2 in [13].

Lemma 4.1 For any $\xi=\left(\xi_{1}, \xi_{2}\right), \eta=\left(\eta_{1}, \eta_{2}\right) \in L_{\Gamma_{1}} \times L_{\Gamma_{2}}$ there exists $\gamma=\left(\gamma_{1}, \gamma_{2}\right) \in$ $\Gamma$ such that $\gamma_{1} \xi_{1} \neq \eta_{1}$ and $\gamma_{2} \xi_{2} \neq \eta_{2}$.

Proof We first treat the case $\xi_{1}=\eta_{1}$ and $\xi_{2} \neq \eta_{2}$. Choose $\gamma=\left(\gamma_{1}, \gamma_{2}\right), \varphi=$ $\left(\varphi_{1}, \varphi_{2}\right) \in \Gamma$ such that $\gamma_{1} \xi_{1} \neq \eta_{1}$ and $\varphi_{2} \xi_{2} \notin\left\{\xi_{2}, \eta_{2}\right\}$. This is possible by Lemma 2.11. If $\gamma_{2} \xi_{2} \neq \eta_{2}, \gamma$ is the desired element, if $\varphi_{1} \xi_{1} \neq \eta_{1}$, then $\varphi$ is.

Suppose now $\gamma_{2} \xi_{2}=\eta_{2}$ and $\varphi_{1} \xi_{1}=\eta_{1}$. Then

$$
\gamma_{1} \varphi_{1} \xi_{1}=\gamma_{1} \eta_{1} \stackrel{\xi_{1}=\eta_{1}}{=} \gamma_{1} \xi_{1} \neq \eta_{1}
$$

by choice of $\gamma$. Moreover, we have $\gamma_{2} \varphi_{2} \xi_{2} \neq \eta_{2}$, because $\gamma_{2} \varphi_{2} \xi_{2}=\eta_{2}=\gamma_{2} \xi_{2}$ implies that $\varphi_{2}=\gamma_{2}^{-1} \gamma_{2} \varphi_{2}$ is contained in the stabilizer of $\xi_{2}$ which is a contradiction to the choice of $\varphi$. Hence $\gamma \varphi$ is the desired element.

If $\xi=\eta$ we choose $\gamma=\left(\gamma_{1}, \gamma_{2}\right) \in \Gamma$ such that $\gamma_{2} \xi_{2} \neq \eta_{2}$ and apply the first case.

Using (10) we immediately obtain the following:

Corollary 4.2 For any regular axial $h \in \Gamma$ and $\xi \in L_{\Gamma_{1}} \times L_{\Gamma_{2}} \subseteq \partial^{F} X$ there exists $\gamma \in \Gamma$ such that $\gamma \xi \in \operatorname{Vis}^{F}\left(h^{+}\right)$.

We now fix a regular axial isometry $h=\left(h_{1}, h_{2}\right) \in \Gamma$ and a base point $o=\left(o_{1}, o_{2}\right) \in X$. The following important theorem implies that $F_{\Gamma}$ can be covered by finitely many $\Gamma$-translates of an appropriate open set in $\partial^{F} X$.

Theorem 4.3 The Furstenberg limit set is minimal, ie $F_{\Gamma}$ is the smallest nonempty, $\Gamma-$ invariant closed subset of $\partial^{F} X$. 
Proof We first show that every nonempty, $\Gamma$-invariant closed subset of $\partial^{F} X$ contains either $h^{+}$or $h^{-}$. Replacing $h$ by its inverse if necessary, it then suffices to prove that $F_{\Gamma}=\overline{\Gamma \cdot h^{+}}$.

Let $A \subseteq \partial^{F} X$ be a nonempty, $\Gamma$-invariant closed set, and $\xi=\left(\xi_{1}, \xi_{2}\right) \in A$. If $\xi \in$ $\left\{h^{+}, h^{-}\right\}$, there is nothing to prove, so assume that there exist indices $i, j \in\{1,2\}$ such that $\xi_{i} \neq h_{i}^{+}$and $\xi_{j} \neq h_{j}^{-}$. If $\xi_{1} \notin\left\{h_{1}^{+}, h_{1}^{-}\right\}$, then - since $\xi_{2}$ is different from at least one of the points $h_{2}^{+}, h_{2}^{-}-$we have $\xi \in \operatorname{Vis}^{F}\left(h^{+}\right)$or $\xi \in \operatorname{Vis}^{F}\left(h^{-}\right)$. So $\lim _{n \rightarrow \infty} h^{-n} \xi=h^{-}$ or $\lim _{n \rightarrow \infty} h^{n} \xi=h^{+}$and we conclude that $h^{+}$or $h^{-}$belongs to $A$. The case $\xi_{2} \notin$ $\left\{h_{2}^{+}, h_{2}^{-}\right\}$is analogous. It therefore remains to consider the possibilities $\xi=\left(h_{1}^{-}, h_{2}^{+}\right)$ or $\xi=\left(h_{1}^{+}, h_{2}^{-}\right)$. In both cases $\xi$ is contained in $L_{\Gamma_{1}} \times L_{\Gamma_{2}}$, so by Corollary 4.2 there exists $\gamma \in \Gamma$ such that $\gamma \xi \in \operatorname{Vis}^{F}\left(h^{+}\right)$. Then $\lim _{n \rightarrow \infty} h^{-n} \gamma \xi=h^{-}$which proves that $h^{-} \in A$.

For the second part of the proof we are going to show the stronger statement that $F_{\Gamma}=\overline{\Gamma \cdot \xi}$ for any $\xi=\left(\xi_{1}, \xi_{2}\right) \in F_{\Gamma}$.

Let $\eta=\left(\eta_{1}, \eta_{2}\right) \in F_{\Gamma}$ arbitrary. If $\eta=\xi$, there is nothing to prove, if $\eta_{1}=\xi_{1}$ or $\eta_{2}=\xi_{2}$, then by Lemma 4.1 there exists $\gamma=\left(\gamma_{1}, \gamma_{2}\right) \in \Gamma$ such that $\gamma_{1} \xi_{1} \neq$ $\eta_{1}$ and $\gamma_{2} \xi_{2} \neq \eta_{2}$. Hence replacing $\xi$ by $\gamma \xi$ if necessary, we may assume that $\eta_{1} \neq \xi_{1}$ and $\eta_{2} \neq \xi_{2}$. Let $U_{1} \subset \partial X_{1}, U_{2} \subset \partial X_{2}$ be neighborhoods of $\eta_{1}, \eta_{2}$ such that $\xi_{1} \notin U_{1}$ and $\xi_{2} \notin U_{2}$, and choose $\tilde{\eta} \in\left(\pi^{F}\right)^{-1}(\eta) \cap L_{\Gamma}$. Then there exists a sequence $\left(\gamma_{n}\right)=\left(\left(\gamma_{n, 1}, \gamma_{n, 2}\right)\right) \subset \Gamma$ such that $\gamma_{n} o \rightarrow \tilde{\eta}=\left(\eta_{1}, \eta_{2}, \theta\right)$ and $\gamma_{n}^{-1} o$ converges as $n \rightarrow \infty$. Since for $i=1,2, d_{i}\left(o_{i}, \gamma_{n, i}^{-1} o_{i}\right)=d_{i}\left(\gamma_{n, i} o_{i}, o_{i}\right) \rightarrow \infty$ we have $\gamma_{n, i}^{-1} o_{i} \rightarrow \zeta_{i} \in \partial X_{i}, i=1,2$, and $\theta\left(o, \gamma_{n}^{-1} o\right)=\theta\left(o, \gamma_{n} o\right) \rightarrow \theta$ as $n \rightarrow \infty$. Hence $\lim _{n \rightarrow \infty} \gamma_{n}^{-1} o \rightarrow \widetilde{\zeta}:=\left(\zeta_{1}, \zeta_{2}, \theta\right) \in \partial X^{\text {reg }}$, and we put $\zeta:=\pi^{F}(\widetilde{\zeta})=\left(\zeta_{1}, \zeta_{2}\right)$.

Moreover, we can assume $\zeta \in \operatorname{Vis}^{F}\left(h^{+}\right)$, because otherwise, by Corollary 4.2, we find $\gamma \in \Gamma$ such that $\gamma \zeta \in \operatorname{Vis}^{F}\left(h^{+}\right)$and we can replace our sequence $\left(\gamma_{n}\right)$ by $\left(\gamma_{n} \gamma^{-1}\right)$.

Let $T \gg 1, \varepsilon>0$ be arbitrary. Then Lemma 2.2 implies the existence of $N \in \mathbb{N}$ such that for all $n \geq N$ and $t \in[0, T]$,

$$
d\left(\sigma_{o_{i}, \gamma_{n, i} o_{i}}(t), \sigma_{o_{i}, \gamma_{n, i} h_{i}^{+}}(t)\right)=d\left(\sigma_{\gamma_{n, i}^{-1} o_{i}, o_{i}}(t), \sigma_{\gamma_{n, i}^{-1} o_{i}, h_{i}^{+}}(t)\right) \leq \frac{\varepsilon}{2}
$$

and $d\left(\sigma_{o_{i}, \gamma_{n, i} o_{i}}(t), \sigma_{o_{i}, \eta_{i}}(t)\right) \leq \varepsilon / 2$. Hence we conclude that as $n \rightarrow \infty \gamma_{n, i} h_{i}^{+} \rightarrow \eta_{i}$ for $i=1,2$, in particular, there exists $\varphi=\left(\varphi_{1}, \varphi_{2}\right) \in \Gamma$ such that $\varphi_{i} h_{i}^{+} \in U_{i}$ and $\varphi_{i} h_{i} \varphi_{i}^{-1}$ is rank one for $i=1,2$.

Assume first that $\xi \in \operatorname{Vis}^{F}\left(\varphi h^{-}\right)$. Then there exists $N \in \mathbb{N}$ such that for $n \geq N$ $\left(\left(\varphi_{1} h_{1} \varphi_{1}^{-1}\right)^{n} \xi_{1},\left(\varphi_{2} h_{2} \varphi_{2}^{-1}\right)^{n} \xi_{2}\right)=\left(\varphi h \varphi^{-1}\right)^{n} \xi \in U_{1} \times U_{2}$.

If $\xi \notin \operatorname{Vis}^{F}\left(\varphi h^{-}\right)$, Corollary 4.2 implies the existence of $\gamma \in \Gamma$ such that $\gamma \xi \in$ $\operatorname{Vis}^{F}\left(\varphi h^{-}\right)$and we conclude $\left(\varphi h \varphi^{-1}\right)^{n} \gamma \xi \in U$ for $n$ sufficiently large. 
Theorem 4.4 The regular geometric limit set $L_{\Gamma} \cap \partial X^{\text {reg }}$ is isomorphic to a product $F_{\Gamma} \times P_{\Gamma}$, where $P_{\Gamma} \subseteq(0, \pi / 2)$ denotes the set of slopes of regular limit points.

Proof If $\widetilde{\xi} \in L_{\Gamma} \cap \partial X^{\text {reg }}$, then $\pi^{F}(\widetilde{\xi}) \in F_{\Gamma}$, and by definition of $P_{\Gamma}$ the slope of $\widetilde{\xi}$ belongs to $P_{\Gamma}$.

Conversely, let $\eta=\left(\eta_{1}, \eta_{2}\right) \in F_{\Gamma}$ and $\theta \in P_{\Gamma}$. We have to show that $\tilde{\eta}:=\left(\eta_{1}, \eta_{2}, \theta\right) \in$ $L_{\Gamma}$. By definition of $P_{\Gamma}$ and Lemma 3.1 there exists a sequence $\left(\gamma_{n}\right) \subset \Gamma$ such that $\theta_{n}:=\theta\left(o, \gamma_{n} o\right)$ converges to $\theta$ as $n \rightarrow \infty$. Moreover, by compactness of $\partial X_{1} \times \partial X_{2}$ a subsequence of $\left(\gamma_{n} o\right)$ converges to a point $\tilde{\xi} \in L_{\Gamma} \cap \partial X^{\text {reg }}$ of slope $\theta$. Put $\xi:=\pi^{F}(\widetilde{\xi})$, and notice that $\tilde{\eta} \in \partial X^{\text {reg }}$ is the unique point in $\left(\pi^{F}\right)^{-1}(\eta)$ of slope $\theta$.

By Theorem $4.3 F_{\Gamma}=\overline{\Gamma \cdot \xi}$ is a minimal closed set under the action of $\Gamma$, hence

$$
\eta \in \overline{\Gamma \cdot \xi}=\pi^{F}(\overline{\Gamma \cdot \widetilde{\xi}}) \text {. }
$$

Since the action of $\Gamma$ on the geometric boundary does not change the slope of a point, we conclude that the closure of $\Gamma \cdot \widetilde{\xi}$ contains $\tilde{\eta}$. In particular $\tilde{\eta} \in \overline{\Gamma \cdot \widetilde{\xi}} \subseteq L_{\Gamma}$.

\section{Density of regular axial isometries}

In this section we will make the stronger assumption that $\Gamma \subset \operatorname{Is}\left(X_{1}\right) \times \operatorname{Is}\left(X_{2}\right)$ acts properly discontinuously on the product $X$ of two locally compact Hadamard spaces $X_{1}, X_{2}$ and contains two isometries $g=\left(g_{1}, g_{2}\right)$ and $h=\left(h_{1}, h_{2}\right)$ such that $g_{1}$ and $h_{1}$ are independent rank one elements of $\Gamma_{1}$ and $g_{2}, h_{2}$ are independent rank one elements in $\Gamma_{2}$. Recall that an isometry $h=\left(h_{1}, h_{2}\right) \in \operatorname{Is}\left(X_{1}\right) \times \operatorname{Is}\left(X_{2}\right)$ is called regular axial if $h_{1}$ and $h_{2}$ are rank one elements. Its attractive fixed point is denoted $\widetilde{h^{+}} \in \partial X^{\text {reg }}$ and we put $h^{+}:=\pi^{F}\left(\widetilde{h^{+}}\right)=\left(h_{1}^{+}, h_{2}^{+}\right)$. Moreover, for $h=\left(h_{1}, h_{2}\right)$ regular axial and $i \in\{1,2\}$ we denote $l_{i}\left(h_{i}\right)$ the translation length of $h_{i}$ in $X_{i}$. The limit cone of $\Gamma$ is defined by

$$
\ell_{\Gamma}:=\overline{\left\{\arctan \left(l_{2}\left(g_{2}\right) / l_{1}\left(g_{1}\right)\right): g=\left(g_{1}, g_{2}\right) \in \Gamma \text { regular axial }\right\}} .
$$

We fix a base point $o=\left(o_{1}, o_{2}\right) \in X$. The following proposition is a key ingredient in the proofs.

Proposition 5.1 Suppose $g=\left(g_{1}, g_{2}\right)$ and $h=\left(h_{1}, h_{2}\right) \in \Gamma$ are regular axial isometries such that $g_{i}$ and $h_{i}$ are independent in $\Gamma_{i}$ for $i=1,2$. Let $\left(\gamma_{n}\right) \subset \Gamma$ be a sequence such that $\gamma_{n} O$ and $\gamma_{n}^{-1} o$ converge to points in $\partial X^{\mathrm{reg}}$ as $n \rightarrow \infty$. Then given arbitrarily small distinct neighborhoods $W_{i}\left(h^{+}\right), W_{i}\left(h^{-}\right) \subset \bar{X}_{i}$ of $h_{i}^{+}, h_{i}^{-}, i=1,2$, there exist $N \in \mathbb{N}, \alpha \in\left\{h^{N}, h^{N} g^{N}, h^{N} g^{-N}\right\}$ and $\beta \in\left\{h^{-N}, h^{-N} g^{N}, h^{-N} g^{-N}\right\}$ such that $\varphi_{n}:=\alpha \gamma_{n} \beta^{-1}$ satisfies $\varphi_{n} o \in W_{1}\left(h^{+}\right) \times W_{2}\left(h^{+}\right)$and $\varphi_{n}^{-1} o \in W_{1}\left(h^{-}\right) \times W_{2}\left(h^{-}\right)$for $n$ sufficiently large. 
Proof For $i=1,2$ and $\eta \in\left\{g^{-}, g^{+}, h^{-}, h^{+}\right\}$let $W_{i}(\eta) \subset \bar{X}_{i}$ be an arbitrary, sufficiently small neighborhood of $\eta_{i}^{+} \in \partial X_{i}$ with $o_{i} \notin W_{i}(\eta)$ such that all $W_{i}(\eta)$ are pairwise disjoint in $\bar{X}_{i}$. According to Lemma 2.6 (c) there exists a constant $N \in \mathbb{N}$ such that for all $\gamma \in\left\{g, g^{-1}, h, h^{-1}\right\}$ and $i \in\{1,2\}$,

$$
\gamma_{i}^{N}\left(\bar{X}_{i} \backslash W_{i}\left(\gamma^{-}\right)\right) \subseteq W_{i}\left(\gamma^{+}\right) .
$$

Denote $F \subset X$ the finite set of points $\left\{o, h^{ \pm N} o, h^{ \pm N} g^{ \pm N} o\right\}$ in $X$. Since $\gamma_{n, i} o_{i}$ converges to a point $\xi_{i} \in \partial X_{i}, i=1,2$, given arbitrary neighborhoods $U_{1} \subset \bar{X}_{1}$, $U_{2} \subset \bar{X}_{2}$ of $\xi_{1}, \xi_{2}$, there exists $N_{+} \in \mathbb{N}$ such that for all $n>N_{+}$and every $x \in F$ we have $\gamma_{n} x \in U_{1} \times U_{2}$. Using the fact that $\bar{X}_{i}=\left(\bar{X}_{i} \backslash W_{i}\left(g^{-}\right)\right) \cup\left(\bar{X}_{i} \backslash W_{i}\left(g^{+}\right)\right)$, and choosing the neighborhoods $U_{i}$ of $\xi_{i}, i \in\{1,2\}$, sufficiently small, we may assume that one of the following six possibilities occurs for all $n>N_{+}$and every $x=\left(x_{1}, x_{2}\right) \in F$ :

Case $1 \gamma_{n, 1} x_{1} \in \bar{X}_{1} \backslash W_{1}\left(h^{-}\right)$and $\gamma_{n, 2} x_{2} \in \bar{X}_{2} \backslash W_{2}\left(h^{-}\right)$.

Then by (11), $h^{N} \gamma_{n} x \in W_{1}\left(h^{+}\right) \times W_{2}\left(h^{+}\right)$.

Case $2 \gamma_{n, 1} x_{1} \in W_{1}\left(h^{-}\right)$and $\gamma_{n, 2} x_{2} \in W_{2}\left(h^{-}\right)$.

Since $W_{i}\left(h^{-}\right) \subset \bar{X}_{i} \backslash W_{i}\left(g^{-}\right), i=1,2$, again by (11), we have $g^{N} \gamma_{n} x \in W_{1}\left(g^{+}\right) \times$ $W_{2}\left(g^{+}\right)$. Hence we are in Case 1 for $g^{N} \gamma_{n} x$, so $h^{N} g^{N} \gamma_{n} x \in W_{1}\left(h^{+}\right) \times W_{2}\left(h^{+}\right)$.

Case $3 \gamma_{n, 1} x_{1} \in W_{1}\left(h^{-}\right)$and $\gamma_{n, 2} x_{2} \in \bar{X}_{2} \backslash\left(W_{2}\left(h^{-}\right) \cup W_{2}\left(g^{-}\right)\right)$.

Then $g^{N} \gamma_{n} x \in W_{1}\left(g^{+}\right) \times W_{2}\left(g^{+}\right)$, which yields $h^{N} g^{N} \gamma_{n} x \in W_{1}\left(h^{+}\right) \times W_{2}\left(h^{+}\right)$.

Case $4 \gamma_{n, 1} x_{1} \in W_{1}\left(h^{-}\right)$and $\gamma_{n, 2} x_{2} \in \bar{X}_{2} \backslash\left(W_{2}\left(h^{-}\right) \cup W_{2}\left(g^{+}\right)\right)$.

Then $g^{-N} \gamma_{n} x \in W_{1}\left(g^{-}\right) \times W_{2}\left(g^{-}\right)$, which gives $h^{N} g^{-N} \gamma_{n} x \in W_{1}\left(h^{+}\right) \times W_{2}\left(h^{+}\right)$.

Case $5 \gamma_{n, 1} x_{1} \in \bar{X}_{1} \backslash\left(W_{1}\left(h^{-}\right) \cup W_{1}\left(g^{-}\right)\right)$and $\gamma_{n, 2} x_{2} \in W_{2}\left(h^{-}\right)$.

Similarly to Case 3 we obtain $h^{N} g^{N} \gamma_{n} x \in W_{1}\left(h^{+}\right) \times W_{2}\left(h^{+}\right)$.

Case $6 \gamma_{n, 1} x_{1} \in \bar{X}_{1} \backslash\left(W_{1}\left(h^{-}\right) \cup W_{1}\left(g^{+}\right)\right)$and $\gamma_{n, 2} x_{2} \in W_{2}\left(h^{-}\right)$.

As in Case 4 we get $h^{N} g^{-N} \gamma_{n} x \in W_{1}\left(h^{+}\right) \times W_{2}\left(h^{+}\right)$.

So we have shown the existence of $\alpha \in\left\{h^{N}, h^{N} g^{N}, h^{N} g^{-N}\right\}$ such that for all $n>N_{+}$ and every $x \in F \quad \alpha \gamma_{n} x \in W_{1}\left(h^{+}\right) \times W_{2}\left(h^{+}\right)$.

A similar case-by-case treatment gives $N_{-} \in \mathbb{N}$ and $\beta \in\left\{h^{-N}, h^{-N} g^{N}, h^{-N} g^{-N}\right\}$ such that $\beta \gamma_{n}^{-1} x \in W_{1}\left(h^{-}\right) \times W_{2}\left(h^{-}\right)$for all $n>N_{-}$and all $x \in F$. Then, putting $\varphi_{n}:=\alpha \gamma_{n} \beta^{-1}$, the claim holds for all $n>\max \left\{N_{+}, N_{-}\right\}$. 
The following theorem relates the limit cone to $P_{\Gamma}$.

Theorem 5.2 If $\Gamma$ contains two regular axial isometries which project to independent rank one elements in each factor then $P_{\Gamma}=\ell_{\Gamma} \cap(0, \pi / 2)$. Moreover, $\ell_{\Gamma}$ is either a point or an interval.

Proof We first prove $\ell_{\Gamma} \cap(0, \pi / 2) \subseteq P_{\Gamma}$ : If $g_{n}=\left(g_{n, 1}, g_{n, 2}\right)$ is a sequence of regular axial isometries such that $\arctan \left(l_{2}\left(g_{n, 2}\right) / l_{1}\left(g_{n, 1}\right)\right)$ converges to $\theta \in(0, \pi / 2)$, we choose

$$
k_{n} \geq 2 n \max \left\{d_{i}\left(o_{i}, \operatorname{Ax}\left(g_{n, i}\right)\right) / l_{i}\left(g_{n, i}\right): i=1,2\right\}
$$

and put $\gamma_{n}:=g_{n}^{k_{n}}$. From

$$
k_{n} l_{i}\left(g_{n, i}\right) \leq d_{i}\left(o_{i}, \gamma_{n, i} o_{i}\right) \leq 2 d_{i}\left(o_{i}, \operatorname{Ax}\left(g_{n, i}\right)\right)+k_{n} l_{i}\left(g_{n, i}\right) \leq k_{n} l_{i}\left(g_{n, i}\right)(1+1 / n)
$$

we get

$$
\begin{aligned}
& \tan \theta=\lim _{n \rightarrow \infty}\left(\frac{l_{2}\left(g_{n, 2}\right)}{l_{1}\left(g_{n, 1}\right)} \cdot\left(1+\frac{1}{n}\right)\right) \geq \lim _{n \rightarrow \infty} \frac{d_{2}\left(o_{2}, \gamma_{n, 2} o_{2}\right)}{d_{1}\left(o_{1}, \gamma_{n, 1} o_{1}\right)}, \\
& \tan \theta=\lim _{n \rightarrow \infty}\left(\frac{l_{2}\left(g_{n, 2}\right)}{l_{1}\left(g_{n, 1}\right)} \cdot \frac{n}{n+1}\right) \leq \lim _{n \rightarrow \infty} \frac{d_{2}\left(o_{2}, \gamma_{n, 2} o_{2}\right)}{d_{1}\left(o_{1}, \gamma_{n, 1} o_{1}\right)},
\end{aligned}
$$

hence the claim.

Let's prove the inclusion $P_{\Gamma} \subseteq \ell_{\Gamma} \cap(0, \pi / 2)$. Denote $g=\left(g_{1}, g_{2}\right), h=\left(h_{1}, h_{2}\right) \in \Gamma$ two regular axial isometries as in Proposition 5.1. For $\eta \in\left\{g^{-}, g^{+}, h^{-}, h^{+}\right\}$and $i \in\{1,2\}$ let $U_{i}(\eta) \subset \bar{X}_{i}$ be a small neighborhood of $\eta_{i}$ with $o_{i} \notin U_{i}(\eta)$ such that all $U_{i}(\eta)$ are pairwise disjoint. Upon taking smaller neighborhoods, Lemma 2.1 provides a constant $c>0$ such that for $i \in\{1,2\}$ any pair of points in distinct neighborhoods can be joined by a rank one geodesic $\sigma_{i} \subset X_{i}$ with $d\left(o_{i}, \sigma_{i}\right) \leq c$. Moreover, according to Lemma 2.7 for $i \in\{1,2\}$ and $\eta \in\left\{h^{-}, h^{+}\right\}$there exist neighborhoods $W_{i}(\eta) \subseteq U_{i}(\eta)$ of $\eta_{i}$ such that every $\gamma=\left(\gamma_{1}, \gamma_{2}\right) \in \Gamma$ with $\gamma_{i} o_{i} \in W_{i}\left(h^{+}\right)$and $\gamma_{i}^{-1} o \in W_{i}\left(h^{-}\right)$, $i=1,2$, is regular axial with $\gamma_{i}^{+} \in U_{i}\left(h^{+}\right)$and $\gamma_{i}^{-} \in U_{i}\left(h^{-}\right), i=1,2$.

Now let $\theta \in P_{\Gamma}$. By definition there exists a sequence $\left(\gamma_{n}\right)=\left(\left(\gamma_{n, 1}, \gamma_{n_{2}}\right)\right) \subset \Gamma$ such that $d_{2}\left(o_{2}, \gamma_{n, 2} o_{2}\right) / d_{1}\left(o_{1}, \gamma_{n, 1} o_{1}\right) \rightarrow \tan \theta, \gamma_{n, 1} o_{1} \rightarrow \xi_{1}, \gamma_{n, 2} o_{2} \rightarrow \xi_{2}$ as $n \rightarrow \infty$. Passing to a subsequence if necessary, we can assume that $\gamma_{n}^{-1} o \rightarrow \widetilde{\zeta}=\left(\zeta_{1}, \zeta_{2}, \theta\right) \in \partial X^{\text {reg }}$ as $n \rightarrow \infty$. By Proposition 5.1 there exist $N_{0} \in \mathbb{N}$, a finite set $\Lambda \subset \Gamma$ and $\alpha, \beta \in \Lambda$ such that for all $n>N_{0}$,

$$
\alpha \gamma_{n} \beta^{-1} o \in W_{1}\left(h^{+}\right) \times W_{2}\left(h^{+}\right) \text {and } \beta \gamma_{n}^{-1} \alpha^{-1} o \in W_{1}\left(h^{-}\right) \times W_{2}\left(h^{-}\right) .
$$


Put $\varphi_{n}:=\alpha \gamma_{n} \beta^{-1}, n \in \mathbb{N}$, and $L:=\max \left\{d_{i}\left(o_{i}, \lambda_{i} o_{i}\right): i \in\{1,2\}, \lambda=\left(\lambda_{1}, \lambda_{2}\right) \in \Lambda\right\}$. Using the triangle inequality we estimate for $i=1,2$

$$
\left|d_{i}\left(o_{i}, \varphi_{n, i} o_{i}\right)-d_{i}\left(o_{i}, \gamma_{n, i} o_{i}\right)\right| \leq 2 L .
$$

Moreover, by choice of the sets $W_{i}\left(h^{ \pm}\right) \subseteq U_{i}\left(h^{ \pm}\right)$we know that for $n>N_{0} \varphi_{n}$ is regular axial with $\varphi_{n}^{+} \in U_{1}\left(h^{+}\right) \times U_{2}\left(h^{+}\right)$and $\varphi_{n}^{-} \in U_{1}\left(h^{-}\right) \times U_{2}\left(h^{-}\right)$. So Lemma 2.1 shows that for $n>N_{0}$ there exists $x_{n, i} \in \operatorname{Ax}\left(\varphi_{n, i}\right)$ such that $d_{i}\left(o_{i}, x_{n, i}\right) \leq c, i=1,2$. We conclude

$$
l_{i}\left(\varphi_{n, i}\right) \leq d_{i}\left(o_{i}, \varphi_{n, i} o_{i}\right) \leq l_{i}\left(\varphi_{n, i}\right)+2 c, \quad i=1,2,
$$

which - together with (12) - implies that $\tan \theta=\lim _{n \rightarrow \infty} l_{2}\left(\varphi_{n, 2}\right) / l_{1}\left(\varphi_{n, 1}\right)$.

Let's prove the last assertion following the lines of the proof of Proposition 2.4 in [13]: If $\ell_{\Gamma}$ is a point, there is nothing to prove. Otherwise we will show that for $\theta, \theta^{\prime} \in$ $\left\{\arctan \left(l_{2}\left(g_{2}\right) / l_{1}\left(g_{1}\right)\right): g=\left(g_{1}, g_{2}\right) \in \Gamma\right.$ regular axial $\}, \theta<\theta^{\prime}$, we have $\left[\theta, \theta^{\prime}\right] \subseteq \ell_{\Gamma}$. Fix $\gamma=\left(\gamma_{1}, \gamma_{2}\right), \varphi=\left(\varphi_{1}, \varphi_{2}\right)$ regular axial such that $\tan \theta=l_{2}\left(\gamma_{2}\right) / l_{1}\left(\gamma_{1}\right)$ and $\tan \theta^{\prime}=l_{2}\left(\varphi_{2}\right) / l_{1}\left(\varphi_{1}\right)$.

Recall that $g, h \in \Gamma$ are two regular axial isometries projecting to independent rank one elements. If $\gamma_{1}, \varphi_{1}$ are not independent, then by Lemma 2.6 (c) there exist $N \in \mathbb{N}, \alpha, \beta \in\left\{h^{N}, h^{-N}, g^{N}, g^{-N}\right\}$ with $\alpha \neq \beta$ such that $\alpha_{1} \gamma_{1} \alpha_{1}^{-1}$ and $\beta_{1} \varphi_{1} \beta_{1}^{-1}$ are independent. Using the fact that the translation length is invariant by conjugation and upon replacing $\gamma$ by $\alpha \gamma \alpha^{-1}$ and $\varphi$ by $\beta \varphi \beta^{-1}$ if necessary, we may assume that $\gamma_{1}$ and $\varphi_{1}$ are independent rank one elements of $\Gamma_{1}$.

Now either $\gamma_{2}$ and $\varphi_{2}$ are independent, or, after replacing $\gamma, \varphi$ by its inverse if necessary, we have $\gamma_{2}^{+}=\varphi_{2}^{+}$. By Proposition 2.8 and Lemma 2.9 there exist $N \in \mathbb{N}$ and $C>0$ such that for $i \in\{1,2\}$ and all $n, m \in \mathbb{N} \backslash\{0\}$,

$$
\begin{gathered}
\left|l_{i}\left(\gamma_{i}^{N n} \varphi_{i}^{N m}\right)-N n l_{i}\left(\gamma_{1}\right)-N m l_{i}\left(\varphi_{1}\right)\right| \leq C . \\
\lim _{k \rightarrow \infty} \frac{l_{2}\left(\gamma_{2}^{N n k} \varphi_{2}^{N m k}\right)}{l_{1}\left(\gamma_{1}^{N n k} \varphi_{1}^{N m k}\right)}=\frac{n l_{2}\left(\gamma_{2}\right)+m l_{2}\left(\varphi_{2}\right)}{n l_{1}\left(\gamma_{1}\right)+m l_{1}\left(\varphi_{1}\right)}, \\
\quad \arctan \left(\frac{l_{2}\left(\gamma_{2}\right)+q l_{2}\left(\varphi_{2}\right)}{l_{1}\left(\gamma_{1}\right)+q l_{1}\left(\varphi_{1}\right)}\right) \in \ell_{\Gamma}
\end{gathered}
$$

Hence

so we have

for every positive rational number $q \in \mathbb{Q}$ and we conclude $\left[\theta, \theta^{\prime}\right] \in \ell_{\Gamma}$.

In order to prove Theorem $\mathrm{D}$ from the introduction, we will need an important definition as a substitute for the more familiar notion of $\Gamma$-duality used eg in $[3 ; 10]$ when dealing with only one factor. 
Definition 5.3 Two points $\xi=\left(\xi_{1}, \xi_{2}\right), \eta=\left(\eta_{1}, \eta_{2}\right) \in \partial^{F} X$ are called $\Gamma$-related if for any neighborhoods $U_{1}, V_{1} \subset \bar{X}_{1}$ of $\xi_{1}, \eta_{1}$ and all neighborhoods $U_{2}, V_{2} \subset \bar{X}_{2}$ of $\xi_{2}, \eta_{2}$ there exists $\gamma=\left(\gamma_{1}, \gamma_{2}\right) \in \Gamma$ such that for $i \in\{1,2\}$,

$$
\gamma_{i}\left(\bar{X}_{i} \backslash U_{i}\right) \subset V_{i}, \quad \gamma_{i}^{-1}\left(\bar{X}_{i} \backslash V_{i}\right) \subset U_{i} .
$$

We will denote $\operatorname{Rel}_{\Gamma}(\xi)$ the set of points in $\partial^{F} X$ which are $\Gamma$-related to $\xi$.

Notice that for any $\xi \in \partial^{F} X$ the set $\operatorname{Rel}_{\Gamma}(\xi)$ is closed with respect to the topology of $\partial^{F} X$. Moreover, if $\eta \in \operatorname{Rel}_{\Gamma}(\xi)$, then $\eta_{1}$ is $\Gamma_{1}-$ dual to $\xi_{1}$ and $\eta_{2}$ is $\Gamma_{2}-$ dual to $\xi_{2}$.

The importance of the notion lies in the following. If $\tilde{h}^{+}, \tilde{h}^{-}$denote the attractive and repulsive fixed point of a regular axial isometry $h=\left(h_{1}, h_{2}\right) \in \Gamma$, then $h^{+}=\pi^{F}\left(\tilde{h}^{+}\right)$ and $h^{-}=\pi^{F}\left(\tilde{h}^{-}\right)$are $\Gamma$-related by Lemma 2.6 (c). Conversely, if $\xi=\left(\xi_{1}, \xi_{2}\right)$, $\eta=\left(\eta_{1}, \eta_{2}\right) \in \partial^{F} X$ are $\Gamma$-related, then by definition there exists a sequence $\left(\gamma_{n}\right)=$ $\left(\left(\gamma_{n, 1}, \gamma_{n, 2}\right)\right) \subset \Gamma$ such that for $i \in\{1,2\}$ we have $\gamma_{n, i} o_{i} \rightarrow \eta_{i}$ and $\gamma_{n, i}^{-1} o_{i} \rightarrow \xi_{i}$ as $n \rightarrow \infty$. Hence if $\xi_{i}$ can be joined to $\eta_{i}$ by a rank one geodesic for $i=1,2$, then in view of Lemma $2.7 \gamma_{n}$ is regular axial for $n$ sufficiently large and satisfies

$$
\gamma_{n, i}^{+} \rightarrow \eta_{i} \quad \text { and } \quad \gamma_{n, i}^{-} \rightarrow \xi_{i} \quad \text { as } n \rightarrow \infty
$$

for $i \in\{1,2\}$. Denote by $\Delta \subset \partial^{F} X \times \partial^{F} X$ the set

$$
\Delta:=\left\{(\xi, \eta) \in \partial^{F} X \times \partial^{F} X: \xi_{1}=\eta_{1} \text { or } \xi_{2}=\eta_{2}\right\} .
$$

Using the above definition, we are now able to prove the following statement which is Theorem D from the introduction and can be viewed as a strong topological version of the double ergodicity property of Poisson boundaries due to Burger and Monod [9] and Kaimanovich [14].

Theorem 5.4 If $\Gamma$ contains two regular axial isometries projecting to independent rank one elements in each factor then the set of pairs of fixed points $\left(\gamma^{+}, \gamma^{-}\right) \subset \partial^{F} X \times \partial^{F} X$ of regular axial isometries $\gamma \in \Gamma$ is dense in $\left(F_{\Gamma} \times F_{\Gamma}\right) \backslash \Delta$.

Proof Denote $g=\left(g_{1}, g_{2}\right)$ and $h=\left(h_{1}, h_{2}\right) \in \Gamma$ two regular axial isometries such that for $i \in\{1,2\} g_{i}$ and $h_{i}$ are independent. In view of the paragraph preceding the theorem we first prove that any two distinct points in $\left\{g^{-}, g^{+}, h^{-}, h^{+}\right\}$are $\Gamma$-related. For $\eta \in\left\{g^{-}, g^{+}, h^{-}, h^{+}\right\}$and $i \in\{1,2\}$ let $U_{i}(\eta) \subset \bar{X}_{i}$ be an arbitrary, sufficiently small neighborhood of $\eta_{i}$ with $o_{i} \notin U_{i}(\eta)$ such that all $U_{i}(\eta)$ are pairwise disjoint. According to Lemma 2.6 (c) there exists a constant $N \in \mathbb{N}$ such that for all $\gamma \in$ $\left\{g, g^{-1}, h, h^{-1}\right\}$ and $i \in\{1,2\}$,

$$
\gamma_{i}^{N}\left(\bar{X}_{i} \backslash U_{i}\left(\gamma^{-}\right)\right) \subseteq U_{i}\left(\gamma^{+}\right)
$$


Let $\gamma, \varphi \in\left\{g, g^{-1}, h, h^{-1}\right\}, \varphi \neq \gamma$. Using the fact that either $\varphi=\gamma^{-1}$ or $\gamma_{i}, \varphi_{i}$ are independent for $i=1,2$ (13) implies

$$
\gamma_{i}^{N} \underbrace{\varphi_{i}^{-N}\left(\bar{X}_{i} \backslash U_{i}\left(\varphi^{+}\right)\right)}_{\subset U_{i}\left(\varphi^{-}\right) \subset \bar{X}_{i} \backslash U_{i}\left(\gamma^{-}\right)} \subset U_{i}\left(\gamma^{+}\right)
$$

and

$$
\left(\gamma_{i}^{N} \varphi_{i}^{-N}\right)^{-1}\left(\bar{X}_{i} \backslash U_{i}\left(\gamma^{+}\right)\right) \subset \varphi_{i}^{N}\left(U_{i}\left(\gamma^{-}\right)\right) \subset U_{i}\left(\varphi^{+}\right)
$$

for $i \in\{1,2\}$. Hence $\varphi^{+} \in \operatorname{Rel}_{\Gamma}\left(\gamma^{+}\right)$.

Next we will show that any $\xi=\left(\xi_{1}, \xi_{2}\right) \in F_{\Gamma}$ with $\xi_{i} \notin\left\{g_{i}^{-}, g_{i}^{+}, h_{i}^{-}, h_{i}^{+}\right\}, i=1,2$, is $\Gamma$-related to any point in $\left\{g^{-}, g^{+}, h^{-}, h^{+}\right\}$. For $\zeta \in\left\{\xi, g^{-}, g^{+}, h^{-}, h^{+}\right\}$and $i \in\{1,2\}$ let $U_{i}(\zeta) \subset \bar{X}_{i}$ be an arbitrary, sufficiently small neighborhood of $\zeta_{i}$ with $o_{i} \notin U_{i}(\zeta)$ such that all $U_{i}(\zeta)$ are pairwise disjoint. By Lemma 2.7 there exist neighborhoods $W_{i}(\zeta) \subseteq U_{i}(\zeta), \zeta \in\left\{\xi, g^{-}, g^{+}, h^{-}, h^{+}\right\}$, such that every $\gamma_{i} \in \Gamma_{i}$ with $\gamma_{i} o_{i} \in W_{i}(\zeta)$, $\gamma_{i}^{-1} o_{i} \in W_{i}(\eta), \eta \in\left\{\xi, g^{-}, g^{+}, h^{-}, h^{+}\right\} \backslash\{\zeta\}$, is rank one with $\gamma_{i}^{+} \in U_{i}(\zeta)$ and $\gamma_{i}^{-} \in U_{i}(\eta)$

Since $\xi \in F_{\Gamma}$, there exists a sequence $\left(\gamma_{n}\right)=\left(\left(\gamma_{n, 1}, \gamma_{n, 2}\right)\right) \subset \Gamma$ such that $\gamma_{n, 1} o_{1} \rightarrow$ $\xi_{1}, \gamma_{n, 2} O_{2} \rightarrow \xi_{2}$. Upon passing to a subsequence if necessary we may assume that $\gamma_{n, 1}^{-1} o_{1} \rightarrow \zeta_{1} \in \partial X_{1}$ and $\gamma_{n, 2}^{-1} o_{2} \rightarrow \zeta_{2} \in \partial X_{2}$. By Proposition 5.1 there exist $N, N_{0} \in \mathbb{N}$ and $\beta \in\left\{h^{-N}, h^{-N} g^{N}, h^{-N} g^{-N}\right\}$ such that for all $n>N_{0} \gamma_{n} \beta^{-1} o \in W_{1}(\xi) \times W_{2}(\xi)$ and $\beta \gamma_{n}^{-1} o \in W_{1}\left(h^{-}\right) \times W_{2}\left(h^{-}\right)$. By Lemma 2.7 we conclude that for $n>N_{0}$ the isometry $\gamma_{n} \beta^{-1}$ is regular axial with $\left(\gamma_{n} \beta^{-1}\right)^{+} \in U_{1}(\xi) \times U_{2}(\xi)$ and $\left(\gamma_{n} \beta^{-1}\right)^{-} \in$ $U_{1}\left(h^{-}\right) \times U_{2}\left(h^{-}\right)$. This implies that $\xi \in \operatorname{Rel}_{\Gamma}\left(h^{-}\right)$and by symmetry

$$
\xi \in \operatorname{Rel}_{\Gamma}\left(g^{-}\right) \cap \operatorname{Rel}_{\Gamma}\left(g^{+}\right) \cap \operatorname{Rel}_{\Gamma}\left(h^{-}\right) \cap \operatorname{Rel}_{\Gamma}\left(h^{+}\right) .
$$

Next we let $\xi=\left(\xi_{1}, \xi_{2}\right), \eta=\left(\eta_{1}, \eta_{2}\right) \in F_{\Gamma}$ such that for $i \in\{1,2\}$ we have $\xi_{i}, \eta_{i} \notin$ $\left\{g_{i}^{-}, g_{i}^{+}, h_{i}^{-}, h_{i}^{+}\right\}$and $\xi_{i} \neq \eta_{i}$. As above, for $\zeta \in\left\{\xi, \eta, h^{-}\right\}$and $i \in\{1,2\}$ let $U_{i}(\zeta) \subset$ $\bar{X}_{i}$ be an arbitrary, sufficiently small neighborhood of $\zeta_{i}$ with $o_{i} \notin U_{i}(\zeta)$ such that all $U_{i}(\zeta)$ are pairwise disjoint. By the arguments in the previous paragraph there exists a regular axial isometry $\varphi \in \Gamma$ with $\varphi^{+} \in U_{1}(\xi) \times U_{2}(\xi)$ and $\varphi^{-} \in U_{1}\left(h^{-}\right) \times U_{2}\left(h^{-}\right)$. In particular, $\varphi_{i}$ and $g_{i}$ are independent for $i=1,2$. Replacing $h$ by $\varphi$ in (14) we know that $\eta \in \operatorname{Rel}_{\Gamma}\left(g^{-}\right) \cap \operatorname{Rel}_{\Gamma}\left(g^{+}\right) \cap \operatorname{Rel}_{\Gamma}\left(\varphi^{-}\right) \cap \operatorname{Rel}_{\Gamma}\left(\varphi^{+}\right)$, in particular $\eta \in \operatorname{Rel}_{\Gamma}\left(\varphi^{+}\right)$. So using the fact that $\eta_{i}$ can be joined to $\varphi_{i}^{+}$by a rank one geodesic in $X_{i}$ for $i=1,2$, given small neighborhoods $U_{i}\left(\varphi^{+}\right) \subseteq U_{i}(\xi)$ for $i \in\{1,2\}$, there exists $\gamma \in \Gamma$ regular axial with $\gamma^{+} \in U_{1}\left(\varphi^{+}\right) \times U_{2}\left(\varphi^{+}\right) \subseteq U_{1}(\xi) \times U_{2}(\xi)$ and $\gamma^{-} \in U_{1}(\eta) \times U_{2}(\eta)$. 


\section{The exponent of growth for a given slope}

For the remainder of the article $X$ is a product of locally compact Hadamard spaces $X_{1}, X_{2}, o=\left(o_{1}, o_{2}\right)$ a fixed base point, and $\Gamma \subset \operatorname{Is}\left(X_{1}\right) \times \operatorname{Is}\left(X_{2}\right)$ a discrete group which contains two isometries $g=\left(g_{1}, g_{2}\right)$ and $h=\left(h_{1}, h_{2}\right)$ such that $g_{i}$ and $h_{i}$ are independent rank one elements of $\Gamma_{i}$ for $i=1,2$. In this section we want to describe the map which assigns to each slope $\theta \in[0, \pi / 2]$ the exponential growth rate of orbit points of $\Gamma$ in $X$ with a prescribed slope $\theta$. Recall the notation introduced in Section 3 and put for $x, y \in X, \theta \in[0, \pi / 2], \varepsilon>0$,

$$
\Gamma(x, y ; \theta, \varepsilon):=\{\gamma \in \Gamma: \gamma y \neq x \text { and }|\theta(x, \gamma y)-\theta|<\varepsilon\} .
$$

For the definition of the exponential growth rate we introduce the following partial sum of the Poincaré series for $\Gamma$ : For $s>0$ we put

$$
Q_{\theta}^{s, \varepsilon}(x, y)=\sum_{\gamma \in \Gamma(x, y ; \theta, \varepsilon)} e^{-s d(x, \gamma y)}
$$

and denote $\delta_{\theta}^{\varepsilon}(x, y)$ its critical exponent, ie the unique real number such that $Q_{\theta}^{s, \varepsilon}(x, y)$ converges if $s>\delta_{\theta}^{\varepsilon}(x, y)$ and diverges if $s<\delta_{\theta}^{\varepsilon}(x, y)$. It is clear that for any $\varepsilon>0$ we have $\delta_{\theta}^{\varepsilon}(x, y) \leq \delta(\Gamma)$, the critical exponent of the Poincare series. Unfortunately, unlike in the case of $\delta(\Gamma)$, where the summation is over all elements in $\Gamma$, this number may depend on $x$ and $y$. If $\varepsilon>\pi / 2$ then the summation above is over all $\gamma \in \Gamma$ with $\gamma y \neq x$. By discreteness of $\Gamma$ we have $\gamma y=x$ for only finitely many $\gamma \in \Gamma$, hence for $\varepsilon>\pi / 2$ we have $\delta_{\theta}^{\varepsilon}(x, y)=\delta(\Gamma)$.

For $n \in \mathbb{N}$ we define

$$
N_{\theta}^{\varepsilon}(x, y ; n):=\#\{\gamma \in \Gamma: n-1<d(x, \gamma y) \leq n,|\theta(x, \gamma y)-\theta|<\varepsilon\},
$$

which can be interpreted as an orbit counting function for orbit points of slope $\varepsilon$-close to $\theta$. Although the proof of the following lemma is standard, we include it here for the convenience of the reader.

Lemma 6.1 We have

$$
\delta_{\theta}^{\varepsilon}(x, y)=\limsup _{n \rightarrow \infty}\left(\log N_{\theta}^{\varepsilon}(x, y ; n)\right) / n .
$$

Proof We clearly have

$$
Q_{\theta}^{s, \varepsilon}(x, y)=\sum_{n=1}^{\infty} \sum_{\substack{\gamma \in \Gamma(x, y ; \theta, \varepsilon) \\ n-1<d(x, \gamma y) \leq n}} e^{-s d(x, \gamma y)},
$$


hence

$$
\begin{aligned}
\sum_{n=1}^{\infty} e^{-s n} N_{\theta}^{\varepsilon}(x, y ; n) & \leq Q_{\theta}^{s, \varepsilon}(x, y) \\
& \leq \sum_{n=1}^{\infty} e^{-s(n-1)} N_{\theta}^{\varepsilon}(x, y ; n)=e^{s} \sum_{n=1}^{\infty} e^{-s n} N_{\theta}^{\varepsilon}(x, y ; n) .
\end{aligned}
$$

Moreover, we can write

$$
e^{-s n} N_{\theta}^{\varepsilon}(x, y ; n)=\left(e^{-s+\left(\log N_{\theta}^{\varepsilon}(x, y ; n)\right) / n}\right)^{n},
$$

so finding an estimate for the term in the bracket independent of $n$ will allow us to compare $Q_{\theta}^{s, \varepsilon}(x, y)$ to a geometric series.

Suppose first that $s>\lim \sup _{n \rightarrow \infty}\left(\log N_{\theta}^{\varepsilon}(x, y ; n)\right) / n$. Then there exists $N \in \mathbb{N}$ such that for any $n \geq N$

$$
\left(\log N_{\theta}^{\varepsilon}(x, y ; n)\right) / n<s
$$

and we estimate

$$
Q_{\theta}^{s, \varepsilon}(x, y) \leq e^{s}\left(\sum_{n=1}^{N-1} e^{-s n} N_{\theta}^{\varepsilon}(x, y ; n)+\sum_{n=N}^{\infty}\left(e^{-s+\left(\log N_{\theta}^{\varepsilon}(x, y ; n)\right) / n}\right)^{n}\right) .
$$

The first sum is finite, and the second term converges because the number inside the brackets is strictly smaller than 1 for all $n \geq N$.

If $s<\limsup _{n \rightarrow \infty}\left(\log N_{\theta}^{\varepsilon}(x, y ; n)\right) / n$, there exists a strictly increasing sequence $\left(n_{k}\right) \subset \mathbb{N}$ such that $\lim _{k \rightarrow \infty}\left(\log N_{\theta}^{\varepsilon}\left(x, y ; n_{k}\right)\right) / n_{k}>s$. In particular there exists $N \in \mathbb{N}$ such that $\left(1 / n_{k}\right) \log N_{\theta}^{\varepsilon}\left(x, y ; n_{k}\right)>s$ for any $k \geq N$. Moreover, since $n_{k} \geq k$ for all $k$, we have

$$
Q_{\theta}^{s, \varepsilon}(x, y) \geq \sum_{k=N}^{\infty}\left(e^{-s+\left(\log N_{\theta}^{\varepsilon}\left(x, y ; n_{k}\right)\right) / n_{k}}\right)^{n_{k}} \geq \sum_{k=N}^{\infty}\left(e^{-s+\left(\log N_{\theta}^{\varepsilon}\left(x, y ; n_{k}\right)\right) / n_{k}}\right)^{k},
$$

which shows that $Q_{\theta}^{s, \varepsilon}(x, y)$ diverges.

Definition 6.2 The number $\delta_{\theta}(\Gamma):=\liminf _{\varepsilon \rightarrow 0} \delta_{\theta}^{\varepsilon}(o, o)$ is called the exponent of growth of $\Gamma$ of slope $\theta$.

The following lemma shows that this number $\delta_{\theta}(\Gamma)$ does not depend on the choice of arguments of $\delta_{\theta}^{\varepsilon}$.

Lemma 6.3 For $x, y \in X$ arbitrary we have $\liminf _{\varepsilon \rightarrow 0} \delta_{\theta}^{\varepsilon}(x, y)=\delta_{\theta}(\Gamma)$. 
Proof Fix $\theta \in[0, \pi / 2]$ and set

$$
H_{\theta}:=\left(\begin{array}{c}
\cos \theta \\
\sin \theta
\end{array}\right)
$$

We first note that for any $x=\left(x_{1}, x_{2}\right), y=\left(y_{1}, y_{2}\right) \in X$ and $\gamma=\left(\gamma_{1}, \gamma_{2}\right) \in \Gamma$ the equality

$$
\left\langle H(x, \gamma y), H_{\theta}\right\rangle=d(x, \gamma y) \cdot \cos (\theta(x, \gamma y)-\theta)
$$

holds. Using

$$
H(x, \gamma y)-H(o, \gamma o)=\left(\begin{array}{l}
d_{1}\left(x_{1}, \gamma_{1} y_{1}\right)-d_{1}\left(o_{1}, \gamma_{1} o_{1}\right) \\
d_{2}\left(x_{2}, \gamma_{2} y_{2}\right)-d_{2}\left(o_{2}, \gamma_{2} o_{2}\right)
\end{array}\right)
$$

setting $c:=d(x, o)+d(y, o)$ and recalling that both $\sin \theta$ and $\cos \theta$ belong to the interval $[0,1]$ we further have

$$
\left|\left\langle H(x, \gamma y)-H(o, \gamma o), H_{\theta}\right\rangle\right| \leq 4 c .
$$

In particular, we conclude

$$
\begin{aligned}
\left\langle H(x, \gamma y), H_{\theta}\right\rangle & \geq\left\langle H(o, \gamma o), H_{\theta}\right\rangle-4 c=d(o, \gamma o) \cdot \cos (\theta(o, \gamma o)-\theta)-4 c \\
& \geq d(x, \gamma y) \cdot \cos (\theta(o, \gamma o)-\theta)-6 c,
\end{aligned}
$$

hence

$$
\cos (\theta(x, \gamma y)-\theta) \geq \cos (\theta(o, \gamma o)-\theta)-\frac{6 c}{d(x, \gamma y)}
$$

This shows that given $\varepsilon>0$, there is $R \gg 1$ such that $d(x, \gamma y)>R$ and $|\theta(o, \gamma o)-\theta|<$ $\varepsilon / 2$ implies $|\theta(x, \gamma y)-\theta|<\varepsilon$. A symmetric argument - with the roles of $(x, \gamma y)$ and $(o, \gamma o)$ exchanged - ensures the existence of $R^{\prime} \gg 1$ such that $d(x, \gamma y)>R^{\prime}$ and $|\theta(x, \gamma y)-\theta|<\varepsilon$ implies $|\theta(o, \gamma o)-\theta|<2 \varepsilon$. Summarizing, we know that for any $\varepsilon>0$ there exists $R \gg 1$ such that for any $\gamma \in \Gamma$ with $d(x, \gamma y)>R$ we have the implications

$$
\gamma \in \Gamma(o, o ; \theta, \varepsilon / 2) \quad \Longrightarrow \quad \gamma \in \Gamma(x, y ; \theta, \varepsilon) \quad \Longrightarrow \quad \gamma \in \Gamma(x, y ; \theta, 2 \varepsilon) .
$$

Since by discreteness of $\Gamma$ there are only finitely many $\gamma \in \Gamma$ with $d(x, \gamma y) \leq R$, we conclude that for any $\varepsilon>0$

$$
\delta_{\theta}^{\varepsilon / 2}(o, o) \leq \delta_{\theta}^{\varepsilon}(x, y) \leq \delta_{\theta}^{2 \varepsilon}(o, o) .
$$

Taking the limit inferior as $\varepsilon$ tends to zero finishes the proof. 
Notice that in the definition of $\delta_{\theta}(\Gamma)$ for $\theta \in(0, \pi / 2)$ one may substitute

$$
\#\left\{\gamma \in \Gamma: \gamma y \neq x, d(x, \gamma y) \leq n,\left|\frac{d_{2}\left(p_{2}(\gamma y), p_{2}(x)\right)}{d_{1}\left(p_{1}(\gamma y), p_{1}(x)\right)}-\tan \theta\right|<\varepsilon\right\}
$$

in Lemma 6.1 instead of $N_{\theta}^{\varepsilon}(x, y ; n)$. Furthermore, the following property holds:

Lemma 6.4 If $L_{\Gamma} \cap \partial X_{\theta} \neq \varnothing$, then $\delta_{\theta}(\Gamma) \geq 0$.

Proof Suppose $L_{\Gamma} \cap \partial X_{\theta} \neq \varnothing$. Then by Lemma 3.1 for any $\varepsilon>0$ there exist infinitely many $\gamma \in \Gamma$ such that $|\theta(o, \gamma o)-\theta|<\varepsilon$. In particular

$$
\sum_{\gamma \in \Gamma(o, o ; \theta, \varepsilon)} 1=Q_{\theta}^{0, \varepsilon}(o, o)
$$

diverges, hence $\delta_{\theta}^{\varepsilon}(o, o) \geq 0$. We conclude $\delta_{\theta}(\Gamma)=\liminf _{\varepsilon \rightarrow 0} \delta_{\theta}^{\varepsilon}(o, o) \geq 0$.

The following proposition states that the map $\theta \mapsto \delta_{\theta}(\Gamma)$ is upper semicontinuous.

Proposition 6.5 Let $\left(\theta_{j}\right) \subset[0, \pi / 2]$ be a sequence converging to $\theta \in[0, \pi / 2]$. Then

$$
\limsup _{j \rightarrow \infty} \delta_{\theta_{j}}(\Gamma) \leq \delta_{\theta}(\Gamma) \text {. }
$$

Proof Let $\varepsilon_{0} \in(0, \pi / 2)$. Then $\theta_{j} \rightarrow \theta$ implies $\left|\theta_{j}-\theta\right|<\varepsilon_{0} / 2$ for $j$ sufficiently large. Let $\varepsilon \in\left(0, \varepsilon_{0} / 2\right)$ and $\gamma \in \Gamma\left(o, o ; \theta_{j}, \varepsilon\right)$. Then

$$
|\theta(o, \gamma o)-\theta|<\varepsilon+\varepsilon_{0} / 2<\varepsilon_{0},
$$

hence for $j$ sufficiently large $\Gamma\left(o, o ; \theta_{j}, \varepsilon\right) \subseteq \Gamma\left(o, o ; \theta, \varepsilon_{0}\right)$. This shows $\delta_{\theta_{j}}^{\varepsilon}(o, o) \leq$ $\delta_{\theta}^{\varepsilon_{0}}(o, o)$, and therefore $\delta_{\theta_{j}}(\Gamma)=\liminf _{\varepsilon \rightarrow 0} \delta_{\theta_{j}}^{\varepsilon}(o, o) \leq \delta_{\theta}^{\varepsilon_{0}}(o, o)$.

We conclude

$$
\limsup _{j \rightarrow \infty} \delta_{\theta_{j}}(\Gamma) \leq \delta_{\theta}^{\varepsilon_{0}}(o, o),
$$

hence $\quad \limsup _{j \rightarrow \infty} \delta_{\theta_{j}}(\Gamma)=\liminf _{\varepsilon_{0} \rightarrow 0}\left(\limsup _{j \rightarrow \infty} \delta_{\theta_{j}}(\Gamma)\right) \leq \liminf _{\varepsilon_{0} \rightarrow 0} \delta_{\theta}^{\varepsilon_{0}}(o, o)=\delta_{\theta}(\Gamma)$.

This completes the proof.

Example Suppose $X$ is a product $X=X_{1} \times X_{2}$ of Hadamard manifolds with pinched negative curvature, and $\Gamma_{1} \subset \operatorname{Is}\left(X_{1}\right), \Gamma_{2} \subset \operatorname{Is}\left(X_{2}\right)$ are convex cocompact groups with critical exponents $\delta_{1}, \delta_{2}$. Then by Theorem 6.2 .5 in [19] there exists a constant $C>1$ such that for all $n \in \mathbb{N}$ we have

$$
\frac{1}{C} e^{\delta_{i} n} \leq \#\left\{\gamma_{i} \in \Gamma_{i}: n-1<d\left(o_{i}, \gamma_{i} o_{i}\right) \leq n\right\} \leq C e^{\delta_{i} n}, \quad i=1,2 .
$$


We are going to examine the action of the product group $\Gamma=\Gamma_{1} \times \Gamma_{2} \subseteq \operatorname{Is}(X)$ on the product manifold $X$. Given $\theta \in(0, \pi / 2)$, we estimate for $\varepsilon>0$ sufficiently small the number of orbit points

$$
\begin{aligned}
\Delta N_{\theta}^{\varepsilon}(o, o ; n)=\#\left\{\gamma=\left(\gamma_{1}, \gamma_{2}\right) \in \Gamma: n-1<\sqrt{d_{1}\left(o_{1}, \gamma_{1} o_{1}\right)^{2}+d_{2}\left(o_{2}, \gamma_{2} o_{2}\right)^{2}} \leq n,\right. \\
\quad|\theta(o, \gamma o)-\theta|<\varepsilon\} \\
\leq \#\left\{\gamma=\left(\gamma_{1}, \gamma_{2}\right) \in \Gamma: n-1<\frac{d_{1}\left(o_{1}, \gamma_{1} o_{1}\right)}{\cos \theta(o, \gamma o)} \leq n,\right. \\
\left.n-1<\frac{d_{2}\left(o_{2}, \gamma_{2} o_{2}\right)}{\sin \theta(o, \gamma o)} \leq n,|\theta(o, \gamma o)-\theta|<\varepsilon\right\} \\
\leq C^{2} \cdot n e^{\delta_{1} n \cos (\theta-\varepsilon)} \cdot e^{\delta_{2} n \sin (\theta+\varepsilon)} .
\end{aligned}
$$

As a lower bound, we obtain

$$
\begin{aligned}
\Delta N_{\theta}^{\varepsilon}(o, o ; n) & \geq \#\left\{\left(\gamma_{1}, \gamma_{2}\right) \in \Gamma: n-1<\frac{d_{1}\left(o_{1}, \gamma_{1} o_{1}\right)}{\cos \theta} \leq n, n-1<\frac{d_{2}\left(o_{2}, \gamma_{2} o_{2}\right)}{\sin \theta} \leq n\right\} \\
& \geq \frac{1}{C^{2}} \cdot e^{\delta_{1} n \cos \theta} \cdot e^{\delta_{2} n \sin \theta}
\end{aligned}
$$

and therefore conclude $\delta_{\theta}(\Gamma)=\delta_{1} \cos \theta+\delta_{2} \sin \theta$. Treating the cases $\theta=0$ and $\theta=\pi / 2$ separately one can easily verify that this equation holds for all $\theta \in[0, \pi / 2]$.

\section{A generic product for $\Gamma$}

Denote $\mathbb{R}_{\geq 0}:=\{t \in \mathbb{R}: t \geq 0\}$. For convenience, we extend the exponent of growth to a map $\Psi_{\Gamma}: \mathbb{R}_{\geq 0}^{2} \rightarrow \mathbb{R}$ as follows: If $x=\left(x_{1}, x_{2}\right) \in \mathbb{R}_{\geq 0}^{2}$ we put $\theta(x):=\arctan \left(x_{2} / x_{1}\right)$ and set

$$
\Psi_{\Gamma}(x):=\|x\| \cdot \delta_{\theta(x)} .
$$

In the remainder of this section we will show that $\Psi_{\Gamma}$ is a concave function, ie for any $x, y \in \mathbb{R}_{\geq 0}^{2}$ and $t \in[0,1]$ we have $\Psi_{\Gamma}(t x+(1-t) y) \geq t \Psi_{\Gamma}(x)+(1-t) \Psi_{\Gamma}(y)$.

Recall that $X$ is a product of locally compact Hadamard spaces $X_{1}, X_{2}, o=\left(o_{1}, o_{2}\right)$ a fixed base point, and $\Gamma \subset \operatorname{Is}\left(X_{1}\right) \times \operatorname{Is}\left(X_{2}\right)$ acts properly discontinuously and contains a pair of isometries $g=\left(g_{1}, g_{2}\right), h=\left(h_{1}, h_{2}\right)$ such that $g_{i}$ and $h_{i}$ are independent rank one elements in $\Gamma_{i}$ for $i=1,2$. Notice that the distance vector $H: X \times X \rightarrow \mathbb{R}^{2}$ defined at the beginning of Section 3 induces a map $\Gamma \rightarrow \mathbb{R}^{2}$ via the assignment $\gamma \mapsto H(o, \gamma o)$. By abuse of notation we will call this map also $H$.

Let $D$ denote the Dirac measure and $v_{\Gamma}:=\sum_{\gamma \in \Gamma} D_{H(\gamma)}$ the counting measure on $\mathbb{R}^{2}$. In a metric space we denote $B(x, r)$ the ball of radius $r \geq 0$ centered at $x$. We will use the following special case of a theorem due to Quint. 
Theorem 7.1 [16, Theorem 3.2.1] If there exist $r, s, c>0$ such that for any $x, y \in \mathbb{R}^{2}$ the inequality

$$
v_{\Gamma}(B(x+y, s)) \geq c \cdot v_{\Gamma}(B(x, r)) \cdot v_{\Gamma}(B(y, r))
$$

holds, then $\Psi_{\Gamma}$ is concave.

In order to prove inequality (17) we will construct a generic product for $\Gamma$ as in [16, Proposition 2.3.1]. The idea behind is to find a finite set in $\Gamma \times \Gamma$ which maps pairs of orbit points $\left(\gamma o, \varphi^{-1} o\right)$ close to a set $\operatorname{Ax}(g)$ or $\operatorname{Ax}(h)$ as in Definition 2.3. Unfortunately, unlike in the case of symmetric spaces, we do not dispose of an equivalent of the result of Abels, Margulis and Soifer [16, Proposition 2.3.4] which plays a crucial role there. Instead, we will exploit the dynamics of a free subgroup of $\langle g, h\rangle \subseteq \Gamma$.

Proposition 7.2 If $\Gamma \subset \operatorname{Is}\left(X_{1}\right) \times \operatorname{Is}\left(X_{2}\right)$ is as above, then there exists a map pr: $\Gamma \times$ $\Gamma \rightarrow \Gamma$ with the following properties:

(a) There exists $\kappa \geq 0$ such that for all $\gamma, \varphi \in \Gamma$ we have

$$
\|H(\operatorname{pr}(\gamma, \varphi))-H(\gamma)-H(\varphi)\| \leq \kappa .
$$

(b) For any $r>0$ there exists a finite set $\Lambda \subset \Gamma$ such that for all $\gamma, \varphi, \hat{\gamma}, \hat{\varphi} \in \Gamma$ with $\|H(\gamma)-H(\hat{\gamma})\| \leq r,\|H(\varphi)-H(\hat{\varphi})\| \leq r$ we have

$$
\operatorname{pr}(\gamma, \varphi)=\operatorname{pr}(\hat{\gamma}, \hat{\varphi}) \quad \Longrightarrow \quad \hat{\gamma} \in \gamma \Lambda \text { and } \hat{\varphi} \in \Lambda \varphi \text {. }
$$

Proof For $\eta \in\left\{g^{-}, g^{+}, h^{-}, h^{+}\right\}$and $i \in\{1,2\}$ let $U_{i}(\eta) \subset \bar{X}_{i}$ be a small neighborhood of $\eta_{i}$ with $o_{i} \notin U_{i}(\eta)$ such that all $U_{i}(\eta)$ are pairwise disjoint. Upon taking smaller neighborhoods, Lemma 2.1 provides a constant $c>0$ such that for $i \in\{1,2\}$ any pair of points in distinct neighborhoods can be joined by a rank one geodesic $\sigma_{i} \subset X_{i}$ with $d\left(o_{i}, \sigma_{i}\right) \leq c$.

In order to construct a map satisfying property (a) we let $\gamma=\left(\gamma_{1}, \gamma_{2}\right), \varphi=\left(\varphi_{1}, \varphi_{2}\right) \in \Gamma$ arbitrary. Arguing as in the proof of Proposition 5.1 there exist a finite set $\Lambda \subset \Gamma$ and $\alpha=\alpha(\varphi), \beta=\beta(\gamma) \in \Lambda$ such that

$$
\beta \gamma^{-1} o \in U_{1}\left(h^{-}\right) \times U_{2}\left(h^{-}\right) \text {and } \alpha \varphi o \in U_{1}\left(h^{+}\right) \times U_{2}\left(h^{+}\right) .
$$

As in the proof of Theorem 5.2 we set $L:=\max \left\{d_{i}\left(o_{i}, \lambda_{i} o_{i}\right): i \in\{1,2\}, \lambda \in \Lambda\right\}$. For $i=1,2$ we choose a point $x_{i}$ on the geodesic joining $\beta_{i} \gamma_{i}^{-1} o_{i}$ to $\alpha_{i} \varphi_{i} o_{i}$ with $d_{i}\left(o_{i}, x_{i}\right) \leq c$. Then

$$
d_{i}\left(\gamma_{i} \beta_{i}^{-1} \alpha_{i} \varphi_{i} o_{i}, o_{i}\right)=d_{i}\left(\alpha_{i} \varphi_{i} o_{i}, \beta_{i} \gamma_{i}^{-1} o_{i}\right)=d_{i}\left(\alpha_{i} \varphi_{i} o_{i}, x_{i}\right)+d_{i}\left(x_{i}, \beta_{i} \gamma_{i}^{-1} o_{i}\right)
$$


and we can estimate

$$
\begin{aligned}
d_{i}\left(\gamma_{i} \beta_{i}^{-1} \alpha_{i} \varphi_{i} o_{i}, o_{i}\right) \leq & d_{i}\left(\alpha_{i} \varphi_{i} o_{i}, \alpha_{i} o_{i}\right)+\overbrace{d_{i}\left(\alpha_{i} o_{i}, o_{i}\right)}^{\leq L}+\overbrace{d_{i}\left(o_{i}, x_{i}\right)}^{\leq c} \\
& \quad+d_{i}\left(x_{i}, o_{i}\right)+d_{i}\left(o_{i}, \beta_{i} o_{i}\right)+d_{i}\left(\beta_{i} o_{i}, \beta_{i} \gamma_{i}^{-1} o_{i}\right) \\
\leq & d_{i}\left(\varphi_{i} o_{i}, o_{i}\right)+d_{i}\left(\gamma_{i} o_{i}, o_{i}\right)+2 c+2 L
\end{aligned}
$$

and

$$
d_{i}\left(\gamma_{i} \beta_{i}^{-1} \alpha_{i} \varphi_{i} o_{i}, o_{i}\right) \geq d_{i}\left(\varphi_{i} o_{i}, o_{i}\right)+d_{i}\left(\gamma_{i} o_{i}, o_{i}\right)-2 c-2 L .
$$

This gives

$$
\left\|H\left(\gamma \beta^{-1} \alpha \varphi\right)-H(\varphi)-H(\gamma)\right\| \leq 2 \sqrt{2}(c+L)=: \kappa,
$$

hence the assignment $\operatorname{pr}(\gamma, \varphi):=\gamma \beta(\gamma)^{-1} \alpha(\varphi) \varphi$ satisfies property (a).

It remains to prove that the map pr from above also satisfies property (b). Suppose there exists $r>0$ such that for any finite set $\Lambda_{n} \subseteq\{\gamma \in \Gamma: d(o, \gamma o) \leq n\}$ with $n \in \mathbb{N}$ there exist $\gamma_{n}, \varphi_{n}, \hat{\gamma}_{n}, \hat{\varphi}_{n}$ with $\left\|H\left(\gamma_{n}\right)-H\left(\hat{\gamma}_{n}\right)\right\| \leq r,\left\|H\left(\varphi_{n}\right)-H\left(\hat{\varphi}_{n}\right)\right\| \leq r$ and $g_{n}:=\operatorname{pr}\left(\gamma_{n}, \varphi_{n}\right)=\operatorname{pr}\left(\hat{\gamma}_{n}, \hat{\varphi}_{n}\right)$, but $\gamma_{n}^{-1} \hat{\gamma}_{n} \notin \Lambda_{n}$ or $\hat{\varphi}_{n} \varphi_{n}^{-1} \notin \Lambda_{n}$.

Passing to a subsequence if necessary we may assume that all the sequences $\left(\gamma_{n}^{-1} o\right)$, $\left(\hat{\gamma}_{n}^{-1} o\right),\left(\varphi_{n} o\right),\left(\hat{\varphi}_{n} o\right) \subset X$ converge. Notice that even though one of the projections of the sequences to $X_{1}$ or $X_{2}$ may not converge to a boundary point, the arguments from the proof of Proposition 5.1 show that there exist a finite set $\Lambda \subset \Gamma$ and $\alpha, \hat{\alpha}, \beta$, $\widehat{\beta} \in \Lambda$ such that for all $n \in \mathbb{N}$

$$
\beta \gamma_{n}^{-1} o, \hat{\beta} \widehat{\gamma}_{n}^{-1} o \in U_{1}\left(h^{-}\right) \times U_{2}\left(h^{-}\right) \quad \text { and } \quad \alpha \varphi_{n} o, \widehat{\alpha} \hat{\varphi}_{n} o \in U_{1}\left(h^{+}\right) \times U_{2}\left(h^{+}\right) .
$$

For $n \in \mathbb{N}$ and $i=1,2$ we denote $x_{n, i}$ a point on the geodesic path from $\beta_{i} \gamma_{n, i}^{-1} o_{i}$ to $\alpha_{i} \varphi_{n, i} o_{i}$, and $\hat{x}_{n, i}$ a point on the geodesic path from $\widehat{\beta}_{i} \widehat{\gamma}_{n, i}^{-1} o_{i}$ to $\widehat{\alpha}_{i} \widehat{\varphi}_{n, i} o_{i}$ such that $d_{i}\left(o_{i}, x_{n, i}\right) \leq c$ and $d_{i}\left(o_{i}, \hat{x}_{n, i}\right) \leq c$. Furthermore, using $g_{n}=\gamma_{n} \beta^{-1} \alpha \varphi_{n}=\widehat{\gamma}_{n} \hat{\beta}^{-1} \widehat{\alpha} \hat{\varphi}_{n}$ and denoting for $i=1,2 \sigma_{n, i}$ the geodesic path $\sigma_{o_{i}, g_{n, i} o_{i}}$ there exist $t_{i}, \widehat{t}_{i}>0$ such that

$$
\begin{aligned}
& d_{i}\left(\gamma_{n, i} \beta_{i}^{-1} o_{i}, \sigma_{n, i}\left(t_{i}\right)\right)=d_{i}\left(\gamma_{n, i} \beta_{i}^{-1} o_{i}, \sigma_{n, i}\right)=d_{i}\left(o_{i}, \beta_{i} \gamma_{n, i}^{-1} \sigma_{n, i}\right)=d_{i}\left(o_{i}, x_{n, i}\right) \leq c, \\
& d_{i}\left(\hat{\gamma}_{n, i} \hat{\beta}_{i}^{-1} o_{i}, \sigma_{n, i}\left(\hat{t}_{i}\right)\right)=d_{i}\left(\widehat{\gamma}_{n, i} \hat{\beta}_{i}^{-1} o_{i}, \sigma_{n, i}\right)=d_{i}\left(o_{i}, \hat{\beta}_{i} \hat{\gamma}_{n, i}^{-1} \sigma_{n, i}\right)=d_{i}\left(o_{i}, \hat{x}_{n, i}\right) \leq c .
\end{aligned}
$$

by (18) and Lemma 2.1. Hence using $L:=\max \left\{d_{i}\left(o_{i}, \lambda_{i} o_{i}\right): i \in\{1,2\}, \lambda \in \Lambda\right\}$

$$
\begin{aligned}
& d_{i}\left(\gamma_{n, i} o_{i}, \sigma_{n, i}\right) \leq d_{i}\left(\gamma_{n, i} o_{i}, \gamma_{n, i} \beta_{i}^{-1} o_{i}\right)+d_{i}\left(\gamma_{n, i} \beta_{i}^{-1} o_{i}, \sigma_{n, i}\left(t_{i}\right)\right) \leq L+c, \\
& d_{i}\left(\hat{\gamma}_{n, i} o_{i}, \sigma_{n, i}\right) \leq d_{i}\left(\hat{\gamma}_{n, i} o_{i}, \hat{\gamma}_{n, i} \hat{\beta}_{i}^{-1} o_{i}\right)+d_{i}\left(\widehat{\gamma}_{n, i} \hat{\beta}_{i}^{-1} o_{i}, \sigma_{n, i}\left(\hat{t}_{i}\right)\right) \leq L+c .
\end{aligned}
$$

For $n \in \mathbb{N}$ and $i=1,2$ let $y_{n, i}, \hat{y}_{n, i} \in X_{i}$ be the points on the geodesic path $\sigma_{n, i}$ such that $d_{i}\left(o_{i}, y_{n, i}\right)=d_{i}\left(o_{i}, \gamma_{n, i} o_{i}\right)$ and $d_{i}\left(o_{i}, \hat{y}_{n, i}\right)=d_{i}\left(o_{i}, \hat{\gamma}_{n, i} o_{i}\right)$. Since 
$\left\|H\left(\gamma_{n}\right)-H\left(\hat{\gamma}_{n}\right)\right\| \leq r$ we have $d_{i}\left(y_{n, i}, \hat{y}_{n, i}\right) \leq r$, and, by elementary geometric estimates,

$$
d_{i}\left(\gamma_{n, i} o_{i}, y_{n, i}\right) \leq 2(L+c) \quad \text { and } \quad d_{i}\left(\widehat{\gamma}_{n, i} o_{i}, \hat{y}_{n, i}\right) \leq 2(L+c) .
$$

We summarize

$$
\begin{aligned}
d_{i}\left(o_{i}, \gamma_{n, i}^{-1} \hat{\gamma}_{n, i} o_{i}\right) & =d_{i}\left(\gamma_{n, i} o_{i}, \hat{\gamma}_{n, i} o_{i}\right) \\
& \leq d_{i}\left(\gamma_{n, i} o_{i}, y_{n, i}\right)+d\left(y_{n, i}, \hat{y}_{n, i}\right)+d_{i}\left(\hat{y}_{n, i}, \hat{\gamma}_{n, i} o_{i}\right) \\
& \leq 2(L+c)+r+2(L+c), \\
d\left(o, \gamma_{n}^{-1} \hat{\gamma}_{n} o\right) & \leq \sqrt{2}(4 L+4 c+r)=: R .
\end{aligned}
$$

ie

In particular, for $n>R$ we have $\gamma_{n}^{-1} \widehat{\gamma}_{n} \in \Lambda_{n}$, and, in order to obtain the desired contradiction, it remains to prove that $\hat{\varphi}_{n} \varphi_{n}^{-1} \in \Lambda_{n}$ for $n$ sufficiently large.

Notice that $\widehat{\varphi}_{n}=\hat{\alpha}^{-1} \hat{\beta} \hat{\gamma}_{n}^{-1} g_{n}=\hat{\alpha}^{-1} \hat{\beta} \widehat{\gamma}_{n}^{-1} \gamma_{n} \beta^{-1} \alpha \varphi_{n}$, hence

$$
\begin{aligned}
d\left(o, \hat{\varphi}_{n} \varphi_{n}^{-1} o\right)= & d\left(o, \hat{\alpha}^{-1} \hat{\beta} \hat{\gamma}_{n}^{-1} \gamma_{n} \beta^{-1} \alpha o\right) \\
\leq & \overbrace{d\left(o, \hat{\alpha}^{-1} o\right)}^{\leq \sqrt{2} L}+\overbrace{d\left(\hat{\alpha}^{-1} o, \hat{\alpha}^{-1} \hat{\beta} o\right)}^{\leq \sqrt{2} L}+d\left(\hat{\alpha}^{-1} \hat{\beta} o, \widehat{\alpha}{ }^{-1} \hat{\beta} \hat{\gamma}_{n}^{-1} \gamma_{n} o\right) \\
& +d\left(\hat{\gamma}_{n}^{-1} \gamma_{n} o, \hat{\gamma}_{n}^{-1} \gamma_{n} \beta^{-1} o\right)+d\left(\beta^{-1} o, \beta^{-1} \alpha o\right) \\
\leq & d\left(o, \gamma_{n}^{-1} \hat{\gamma}_{n} o\right)+4 \sqrt{2} L \leq R+4 \sqrt{2} L .
\end{aligned}
$$

This finishes the proof.

The following lemma now shows that Equation (17) holds.

Lemma 7.3 There exist $r, s, c>0$ such that for any $x, y \in \mathbb{R}^{2}$ we have

$$
v_{\Gamma}(B(x+y, s)) \geq c \cdot v_{\Gamma}(B(x, r)) \cdot v_{\Gamma}(B(y, r)) .
$$

Proof Notice that $v_{\Gamma}(B(x, r))=\#\{\gamma \in \Gamma:\|H(\gamma)-x\|<r\}$. Fix $r>0$, put $s=\kappa+2 r$ with $\kappa \geq 0$ from Proposition 7.2 (a) and denote $C>0$ the inverse of the cardinality of the set $\Lambda \times \Lambda$ from Proposition 7.2 (b). Put

$$
P(\Gamma):=\{(\gamma, \varphi) \in \Gamma \times \Gamma:\|H(\gamma)-x\|<r,\|H(\varphi)-y\|<r\} .
$$

We will show that for all $x, y \in \mathbb{R}^{2}$

$$
\#\{\gamma \in \Gamma:\|H(\gamma)-x-y\|<s\} \geq C \cdot \# P(\Gamma) .
$$


Let $(\gamma, \varphi) \in P(\Gamma)$. Then $\alpha:=\operatorname{pr}(\gamma, \varphi) \in \Gamma$ satisfies

$$
\begin{aligned}
\|H(\alpha)-x-y\| & \leq\|H(\alpha)-H(\gamma)-H(\varphi)\|+\|H(\gamma)-x\|+\|H(\varphi)-y\| \\
& \leq \kappa+r+r=s .
\end{aligned}
$$

Moreover, Proposition 7.2 (b) implies that the number of different elements in $P(\Gamma)$ which can yield the same element in $\{\gamma \in \Gamma:\|H(\gamma)-x-y\|<s\}$ is bounded by $\#(\Lambda \times \Lambda)$.

As a corollary of Theorem 7.1 and Proposition 7.2 we obtain:

Theorem 7.4 The function $\Psi_{\Gamma}$ is concave.

Together with Proposition 6.5 this gives Theorem E of the introduction.

\section{References}

[1] W Ballmann, Axial isometries of manifolds of nonpositive curvature, Math. Ann. 259 (1982) 131-144 MR656659

[2] W Ballmann, Lectures on spaces of nonpositive curvature, DMV Seminar 25, Birkhäuser Verlag, Basel (1995) MR1377265 With an appendix by M Brin

[3] W Ballmann, M Brin, Orbihedra of nonpositive curvature, Inst. Hautes Études Sci. Publ. Math. (1995) 169-209 (1996) MR1383216

[4] W Ballmann, M Gromov, V Schroeder, Manifolds of nonpositive curvature, Progress in Math. 61, Birkhäuser, Boston (1985) MR823981

[5] Y Benoist, Propriétés asymptotiques des groupes linéaires, Geom. Funct. Anal. 7 (1997) 1-47 MR1437472

[6] M Bestvina, K Fujiwara, A characterization of higher rank symmetric spaces via bounded cohomology, Geom. Funct. Anal. 19 (2009) 11-40 MR2507218

[7] M R Bridson, A Haefliger, Metric spaces of non-positive curvature, Grund. der Math. Wissenschaften 319, Springer, Berlin (1999) MR1744486

[8] M Burger, Intersection, the Manhattan curve, and Patterson-Sullivan theory in rank 2, Internat. Math. Res. Notices (1993) 217-225 MR1230298

[9] M Burger, N Monod, Continuous bounded cohomology and applications to rigidity theory, Geom. Funct. Anal. 12 (2002) 219-280 MR1911660

[10] P-E Caprace, K Fujiwara, Rank one isometries of buildings and quasi-morphisms of Kac-Moody groups arXiv:0809.0470

[11] P-E Caprace, B Rémy, Simplicity and superrigidity of twin building lattices, Invent. Math. 176 (2009) 169-221 MR2485882 
[12] F Dal'Bo, Remarques sur le spectre des longueurs d'une surface et comptages, Bol. Soc. Brasil. Mat. (N.S.) 30 (1999) 199-221 MR1703039

[13] F Dal'Bo, I Kim, Shadow lemma on the product of Hadamard manifolds and applications, from: "Actes du Séminaire de Théorie Spectrale et Géométrie. Vol. 25. Année 2006-2007”, Sémin. Théor. Spectr. Géom. 25, Univ. Grenoble I, Saint (2008) 105-119 MR2478811

[14] V A Kaimanovich, Double ergodicity of the Poisson boundary and applications to bounded cohomology, Geom. Funct. Anal. 13 (2003) 852-861 MR2006560

[15] G Link, Hausdorff dimension of limit sets of discrete subgroups of higher rank Lie groups, Geom. Funct. Anal. 14 (2004) 400-432 MR2062761

[16] J-F Quint, Divergence exponentielle des sous-groupes discrets en rang supérieur, Comment. Math. Helv. 77 (2002) 563-608 MR1933790

[17] J-F Quint, Mesures de Patterson-Sullivan en rang supérieur, Geom. Funct. Anal. 12 (2002) 776-809 MR1935549

[18] B Rémy, Construction de réseaux en théorie de Kac-Moody, C. R. Acad. Sci. Paris Sér. I Math. 329 (1999) 475-478 MR1715140

[19] C Yue, The ergodic theory of discrete isometry groups on manifolds of variable negative curvature, Trans. Amer. Math. Soc. 348 (1996) 4965-5005 MR1348871

Karlsruhe Institute of Technology (KIT)

Kaiserstr 12, 76131 Karlsruhe, Germany

gabriele.link@kit.edu

http: //www.math.kit.edu/iag2/ link

Proposed: Benson Farb

Received: 26 June 2009

Seconded: Martin Bridson, Walter Neumann

Revised: 1 March 2010 